\title{
Bounded size biased couplings, log concave distributions and concentration of measure for occupancy models
}

\author{
JAY BARTROFF ${ }^{1, *}$, LARRY GOLDSTEIN ${ }^{1, * *}$ and ÜMIT IŞLAK ${ }^{2}$ \\ ${ }^{1}$ Department of Mathematics, University of Southern California, Los Angeles, CA 90089, USA. \\ E-mail: *bartroff@usc.edu; ${ }^{* *}$ larry@math.usc.edu \\ ${ }^{2}$ Department of Mathematics, Boğaziçi University, Bebek-Istanbul 34342, Turkey. \\ E-mail: umit.islak1@boun.edu.tr
}

\begin{abstract}
Threshold-type counts based on multivariate occupancy models with log concave marginals admit bounded size biased couplings under weak conditions, leading to new concentration of measure results for random graphs, germ-grain models in stochastic geometry and multinomial allocation models. The results obtained compare favorably with classical methods, including the use of McDiarmid's inequality, negative association, and self bounding functions.
\end{abstract}

Keywords: concentration; coupling; log concave; occupancy; Poisson Binomial distribution

\section{Introduction}

A random graph on $m$ vertices in which edges are independently present between every two distinct vertices is one framework that leads to an occupancy model described by a vector

$$
\mathbf{M}=\left(M_{\alpha}\right)_{\alpha \in[m]}
$$

of nonnegative integer valued random variables $M_{\alpha}$, where $[m]=\{1, \ldots, m\}$ and $M_{\alpha}$ is the degree of vertex $\alpha$. In such models, given a nonnegative integer threshold $d \geq 0$, many authors have studied the distribution of quantities such as

$$
Y_{\mathrm{ge}}=\sum_{\alpha \in[m]} \mathbf{1}\left(M_{\alpha} \geq d\right) \quad \text { and } \quad Y_{\mathrm{eq}}=\sum_{\alpha \in[m]} \mathbf{1}\left(M_{\alpha}=d\right)
$$

which, in the Erdős-Rényi random graph case just described, count the number of vertices that have degree at least and exactly $d$, respectively. Interest in the distributions of the random variables defined in (2) focuses on their approximation by distributional limits such as the normal, and their finite sample concentration properties. The purpose of the current manuscript is the latter, the study of the concentration of such random variables via the use of size biased couplings derived from the Stein's method literature. The concentration of measure phenomenon has received a great deal of attention since the groundbreaking work of Talagrand [53], and has found applications in areas as diverse as statistics, random matrix theory, combinatorics, information theory, and randomized algorithms. We refer to [9] and [37] for excellent treatments of 
the subject. The results in this paper hold for more general occupancy models (1), not just random graphs, and to refer to a generic occupancy model (1) we will call $M_{\alpha}$ a "count" and $\alpha$ an "urn".

To give the flavor of our results, continue to consider the Erdős-Rényi random graph on $m$ vertices where each disjoint pair of vertices is independently connected by an edge with probability $p \in(0,1)$ and $M_{\alpha}$ is the degree of vertex $\alpha$. The work [25] derived concentration results for the number of isolated vertices, or equivalently, for the variable $Y_{\text {ge }}$ in (2) with $d=1$. Here we allow each vertex to have its own threshold $d_{\alpha}$ to either meet, exceed, or differ from, and which are allowed to take any value, each pair of disjoint edges $\{i, j\}$ is to have its own connection probability $p_{\{i, j\}}$, and each vertex to be weighted according to a nonnegative 'importance factor' $w_{\alpha}$. In Theorem 3.1, for random graph models including the Erdôs-Rényi, we provide sub-Poisson concentration bounds for random variables of the form

$$
Y_{\mathrm{ge}}=\sum_{\alpha \in[m]} w_{\alpha} \mathbf{1}\left(M_{\alpha} \geq d_{\alpha}\right) \quad \text { and } \quad Y_{\mathrm{ne}}=\sum_{\alpha \in[m]} w_{\alpha} \mathbf{1}\left(M_{\alpha} \neq d_{\alpha}\right),
$$

that is, for the weighted number of components of $\mathbf{M}$ having size at least $d_{\alpha}$, and not equal to $d_{\alpha}$, respectively. In addition,

1. For the germ-grain models in stochastic geometry introduced in Section 3.2, Theorems 3.2 and 3.3 provide sub-Poisson concentration results for the volume covered by multi-way intersections, and counts of neighbors, respectively.

2. For the multinomial model introduced in Section 3.3, Theorem 3.4 provides sub-Poisson concentration results for urn occupancy counts.

The current work springs from that of [23] and [24], which demonstrated how bounded size bias couplings can be used to achieve concentration of measure results. Those works in turn were built on the base of [12], which showed how tools from Stein's method (see [51] and [52], and [13] and [46] for overviews), and in particular exchangeable pairs, can be used to expand the scope of application of the concentration of measure phenomenon. Through the use of bounded size bias couplings, [24] produced concentration results for examples including the number of relatively ordered subsequences of a random permutation, the number of local maxima of a random function on a lattice, the number of urns containing exactly one ball in a uniform urn allocation model, and the volume covered by the union of $n$ balls placed uniformly over a subset of $\mathbb{R}^{p}$ with volume $n$. In [25], a concentration result was obtained for the number of isolated vertices in the Erdős-Rényi random graph.

Lemma 2.1, one main result in the present work, provides a framework for the construction of bounded size bias couplings for threshold counts of random variables having a discrete $\log$ concave distribution. Such constructions allow the results of [24] and [25] to be extended to counts of multinomial urn occupancies that exceed or meet any values, to the covered volume of multi-way intersections in germ-grain models, and to counts of the number of vertices of the Erdős-Rényi graph having any degrees. Further, we do not require an identical distribution assumption and consider occupancy thresholds and importance weightings that may depend on the component $\alpha \in[m]$. In Section 4, we show how our results improve on what can be obtained by competing methods. 
In order to prepare for the rest of the paper, we provide some background on size biased distributions and couplings. First, recall that for a nonnegative random variable $Y$ with finite positive mean $\mu$, we say that $Y^{s}$ has the $Y$-size bias distribution if

$$
E[Y f(Y)]=\mu E\left[f\left(Y^{s}\right)\right]
$$

for all functions $f$ for which these expectations exist. For a survey on the diverse appearances of size biasing in probability and statistics, see [4]. We say we have a size bias coupling when a random variable $Y^{s}$ satisfying (4) is defined on the same space as $Y$, and the coupling is said to be bounded when there exists $c \in[0, \infty)$ such that $\left|Y^{s}-Y\right| \leq c$ almost surely. The work [24] showed that for nonnegative $Y$ with finite mean $\mu$ and bounded size bias coupling $Y^{s}$ satisfying $\left|Y^{s}-Y\right| \leq c$, if $Y^{s} \geq Y$ then

$$
P(Y-\mu \leq-t) \leq \exp \left(-\frac{t^{2}}{2 c \mu}\right) \quad \text { for all } t>0 .
$$

And if the moment generating function $m(\theta)=E\left(e^{\theta Y}\right)$ is finite at $\theta=2 / c$, then

$$
P(Y-\mu \geq t) \leq \exp \left(-\frac{t^{2}}{2 c \mu+c t}\right) \quad \text { for all } t>0 .
$$

The bound (5) holds without the monotonicity assumption and we prove this in the Appendix, thus providing a left tail bound for any application in [24] which previously lacked one. After a version of this manuscript was circulated, Theorem 1.1 below of [3] removed the monotonicity assumption using different methods, and further improved the result of [24] by removing the assumption that the moment generating function of $Y$ be finite at $2 / c$, relaxing the bounded coupling condition to $Y^{s}-Y \leq c$, and by improving the inequality to (7) which, as shown there, implies (5) and (6).

The subsequent work [16] strictly generalizes Theorem 1.1 of [3] by, in Theorems 3.3 and 3.4, relaxing the almost sure boundedness assumption by the condition that there exists $p \in(0,1]$ such that $P\left[X^{s} \leq X+c \mid X^{s} \geq x\right] \geq p$ for all $x$ for an upper tail bound, and $P\left[X^{s} \leq X+c \mid X \leq\right.$ $x] \geq p$ for all $x$ for the lower tail. Theorem 3.4 is in the spirit of Bennett's inequality, with upper bounds given in terms of a variance proxy, rather than the mean.

Theorem 1.1. Let $Y$ be a nonnegative random variable with nonzero, finite mean $\mu$, and suppose there exists a coupling of $Y$ to a variable $Y^{s}$ having the $Y$-size bias distribution that satisfies $Y^{s} \leq Y+c$ for some $c>0$ with probability one. Then

$$
\max \left\{\sup _{t \geq 0} P(Y-\mu \geq t), \sup _{-\mu \leq t \leq 0} P(Y-\mu \leq t)\right\} \leq\left(\frac{\mu}{\mu+t}\right)^{(t+\mu) / c} e^{t / c} .
$$

Note that the upper tail inequality given in (7) can be rewritten in the more familiar form

$$
P(Y-\mu \geq t) \leq \exp \left(-\frac{\mu}{c} h\left(\frac{t}{\mu}\right)\right) \quad \text { for all } t>0,
$$


where $h(x)=(1+x) \log (1+x)-x, x \geq-1$. Using the inequality

$$
h(x) \geq \frac{x^{2}}{2+2 x / 3}, \quad x \geq 0
$$

(e.g., see [9], Exercise 2.8), one immediately obtains the following Bernstein type inequality as a corollary, which provides a slight improvement over (6).

Corollary 1.1. In the setting of Theorem 1.1,

$$
P(Y-\mu \geq t) \leq \exp \left(-\frac{t^{2}}{2 c \mu+2 c t / 3}\right) \quad \text { for all } t>0 .
$$

Next, we briefly review constructions of random variables having the size bias distribution of linear combinations of indicator random variables. Throughout we will write $\mathcal{L}(\cdot)$ for law, or distribution.

We start by stating Lemma 4.1 of [27]. When $A$ is an event satisfying $0<P(A)<1$ and $\mathcal{F}$ is a $\sigma$-algebra, a simple application of nested conditioning shows that for all bounded continuous functions $f$, the random variable ${ }^{1} Y=P(A \mid \mathcal{F})$ satisfies

$$
E(Y f(Y))=E\left(f(Y) \mathbf{1}_{A}\right)=\mathbb{P}(A) \mathbb{E}(f(Y) \mid A) \quad \text { and hence } \quad \mathcal{L}(Y \mid A)=\mathcal{L}\left(Y^{s}\right) .
$$

Next, Lemma 1.1 is a special case of a result of [28] that suggests constructions of size biased couplings for sums of nonnegative random variables with finite means.

Lemma 1.1. Let $Y=\sum_{\alpha \in[m]} w_{\alpha} X_{\alpha}$ be a sum of Bernoulli variables $\left(X_{\alpha}\right)_{\alpha \in[m]}$ weighted by nonnegative constants $\left(w_{\alpha}\right)_{\alpha \in[m]}$ and satisfying $E Y>0$. Suppose that for each $\alpha \in[m]$ the variables $\left\{X_{\beta}^{\alpha}, \beta \in[m]\right\}$ are defined on a common probability space such that

$$
\mathcal{L}\left(X_{\beta}^{\alpha}, \beta \in[m]\right)=\mathcal{L}\left(X_{\beta}, \beta \in[m] \mid X_{\alpha}=1\right) .
$$

Then for each $\alpha \in[m]$ letting

$$
Y^{\alpha}=\sum_{\beta \in[m]} w_{\beta} X_{\beta}^{\alpha},
$$

and I a random index with distribution

$$
P(I=\alpha)=\frac{w_{\alpha} E X_{\alpha}}{E Y}
$$

the law $\mathcal{L}\left(Y^{I}\right)$ given by the mixture $\sum_{\alpha \in[m]} P(I=\alpha) \mathcal{L}\left(Y^{\alpha}\right)$ is the $Y$-size bias distribution.

${ }^{1}$ In (9) and below, for an event $A$ we write $\mathcal{L}(Y \mid A)$ to denote the law of the random variable with distribution $P(Y \in$ $B \mid A)$. We abuse notation in the standard way by writing $P(\cdot \mid \mathcal{F})$ and $P(\cdot \mid R)$ to denote conditioning on a $\sigma$-algebra $\mathcal{F}$ and the $\sigma$-algebra $\sigma(R)$ generated by a random variable $R$. 
We note that $\left(X_{\alpha}\right)_{\alpha \in[m]}$ is allowed to have any joint distribution with Bernoulli marginals. In addition, the joint distributions of $\left\{X_{\beta}^{\alpha}, \beta \in[m]\right\}$ are constrained only to satisfy (10), and in particular, if all variables are defined on a common space then the dependence structure between the collections $\left(X_{\beta}^{\alpha}\right)_{\beta \in[m]}$ over $\alpha \in[m]$ can be arbitrary. In this latter case, and when $I$ is defined on the common space, we construct $I$ independent of $\left(X_{\beta}^{\alpha}\right)_{\beta \in[m]}$ so that $E g\left(Y^{I}\right)=\sum_{\alpha \in[m]} P(I=\alpha) E\left(g\left(Y^{\alpha}\right) \mid I=\alpha\right)=\sum_{\alpha \in[m]} P(I=\alpha) E\left(g\left(Y^{\alpha}\right)\right)$, thus achieving the desired mixture of the lemma.

To understand the connections between (9) and Lemma 1.1, let us briefly explain how the former implies the latter. Suppose $Y$ is given as the weighted sum of Bernoulli variables, as in Lemma 1.1. Then letting $w=\sum_{\alpha \in[m]} w_{\alpha}$, for an index $J$ with distribution $P(J=\alpha)=w_{\alpha} / w$, $\alpha \in[m]$, chosen independently of $\left(X_{\alpha}\right)_{\alpha \in[m]}$, and $A=\left\{X_{J}=1\right\}$ and $\mathcal{F}=\sigma\left\{X_{\alpha}, \alpha \in[m]\right\}$ we obtain

$$
w P(A \mid \mathcal{F})=\sum_{\alpha \in[m]} w_{\alpha} X_{\alpha}=Y .
$$

Taking expectation in (13) yields $w P(A)=E Y$. Now, if $\mathcal{L}\left(Y^{\prime}\right)=\mathcal{L}(Y \mid A)$ then, with $Y^{\alpha}$ as in (11) and $I$ with distribution (12), the reader can easily check that $E\left[g\left(Y^{I}\right)\right]=E\left[g\left(Y^{\prime}\right)\right]$ for all bounded continuous functions $g$ so that, by $(9), \mathcal{L}\left(Y^{I}\right)$ of Lemma 1.1 is the $Y$-size bias distribution.

The rest of the paper is organized as follows. Section 2 shows how to construct bounded size bias couplings for random variables of the form (3) when the components $M_{\alpha}$ of $\mathbf{M}$ have a discrete $\log$ concave distribution, with support bounded from below in the case of $Y_{\text {ge }}$, and when for all $\alpha \in[m]$ the remaining counts conditioned on $M_{\alpha}=a$ and $M_{\alpha}=b$ for $a, b \in \mathcal{S}_{\alpha}$ can be closely coupled whenever $a$ is 'close' to $b$. In Section 3, we provide complete descriptions of the three models mentioned above, and apply the results of Section 2 to obtain concentration of measure inequalities. A comparison of the size bias method for concentration with other techniques in the literature is included in Section 4. The Appendix contains the proof that (5) holds without the monotonicity assumption $Y^{s} \geq Y$.

\section{Bounded coupling constructions under log concavity}

The purpose of this section is to form the theoretical background for size biased coupling constructions that are to be used for obtaining concentration of measure inequalities for the statistics described in the Introduction. First, we note that Lemma 1.1 gives a recipe for the construction of a variable having the $Y$-size biased distribution, and in particular only suggests how a coupling may be created. Here, we construct couplings not directly on the occupancy vectors $\mathbf{M}=\left(M_{\alpha}\right)_{\alpha \in[m]}$ themselves, but on a collection of 'more basic' variables $\mathcal{U}$ that we term configurations, and of which the occupancy counts are functions. The configuration $\mathcal{U}$ will be specified for each application. For instance, when $\mathbf{M}$ is the count of vertex degrees in an Erdôs-Rényi graph on a vertex set $[m]$ we take the configuration $\mathcal{U}$ to be the collection of edge indicator variables $\left(X_{\{\alpha, \beta\}}\right)_{\alpha \neq \beta,\{\alpha, \beta\} \subset[m]}$, and, similarly, when $\mathbf{M}$ counts the number of balls in each urn in a multinomial model, the configuration $\mathcal{U}$ records the location of each ball. In these examples, the variables making up the configurations $\mathcal{U}$ are random, but we also will refer to realizations of $\mathcal{U}$ as configurations, so configurations may also contain deterministic variables. 
Definition 2.1. When the occupancy counts $\mathbf{M}$ are given as $F(\mathcal{U})$ for some collection of (possibly random) variables $\mathcal{U}$ and measurable function $F$, we say that $\mathbf{M}$ corresponds to the configuration $\mathcal{U}$ through $F$, or that configuration $\mathcal{U}$ has corresponding occupancy counts $\mathbf{M}$ with respect to $F$.

The function $F$ will be fixed in each of our applications, and so we may omit its mention when there is no possibility of confusion. In such cases we may write, for instance, that $\mathcal{U}$ has corresponding occupancy counts $\mathbf{M}$.

Specializing to the case of interest here, Lemma 1.1 suggests the following size bias coupling construction for sums of the form (3), say $Y_{\text {ge }}$ for concreteness. Following (10), given a configuration $\mathcal{U}$ from the model, one constructs $\mathcal{U}^{\alpha}$, one for each $\alpha \in[m]$, on the same space as $\mathcal{U}$, with law given by

$$
\mathcal{L}\left(\mathcal{U}^{\alpha}\right)=\mathcal{L}\left(\mathcal{U} \mid X_{\alpha}=1\right)=\mathcal{L}\left(\mathcal{U} \mid M_{\alpha} \geq d_{\alpha}\right) .
$$

One then obtains the variable $Y_{\mathrm{ge}}^{\alpha}$ by evaluating the sum $Y_{\mathrm{ge}}$ of (3) on the occupancy counts corresponding to $\mathcal{U}^{\alpha}$, and the size bias variable $Y_{\mathrm{ge}}^{s}$ by selecting $Y^{\alpha}$ with probability proportional to the expectation of $w_{\alpha} X_{\alpha}=w_{\alpha} \mathbf{1}\left(M_{\alpha} \geq d_{\alpha}\right)$, independently of all else.

For the construction of a configuration satisfying (14), Lemma 2.1 and Corollary 2.1 below show how to achieve a bounded coupling between $M_{\alpha}$ and a variable with distribution $\mathcal{L}\left(M_{\alpha} \mid M_{\alpha} \geq d_{\alpha}\right)$ when the distribution of $M_{\alpha}$ is $\log$ concave. Lemma 2.4 will be used to construct the remainder of a configuration that has the correct conditional counts for the urns $\beta \neq \alpha$ when their marginals have the distribution of the sum of independent Bernoulli variables, that is, when they have a Poisson Binomial distribution [29]; see (22) for a formal definition.

For any nonnegative integer $m$ let $[m]_{0}=\{0, \ldots, m\}$, and for any subset $\mathcal{S}$ of $\mathbb{R}$ and any $t_{1}, t_{2} \in \mathbb{R}$, let $t_{1} \mathcal{S}+t_{2}=\left\{t_{1} s+t_{2}: s \in \mathcal{S}\right\}$. For instance, $[m]-1=[m-1]_{0}$ when $m \geq 1$. For a discrete random variable $M$, let $p_{x}=P(M=x)$ and $\operatorname{supp}(M)=\left\{x \in \mathbb{R}: p_{x}>0\right\}$ be the probability mass function and support of $M$, respectively. Recall that $M$ is called a lattice random variable if $\operatorname{supp}(M) \subset h_{1} \mathbb{Z}+h_{2}$ for some real numbers $h_{1} \neq 0, h_{2}$. We can without loss of generality assume our lattice random variables $M$ have $\operatorname{supp}(M) \subset \mathbb{Z}$ by applying the transformation $\left(M-h_{2}\right) / h_{1}$. Such a lattice random variable $M$ is log concave $(L C)$ if supp $(M)$ is an integer interval; that is, if

$$
\operatorname{supp}(M)=\left(k_{1}, k_{2}\right) \cap \mathbb{Z} \quad \text { for some } k_{1}, k_{2} \in \mathbb{Z} \cup\{ \pm \infty\}, k_{1}<k_{2}-1,
$$

and

$$
p_{x}^{2} \geq p_{x-1} p_{x+1} \quad \text { for all } x \in \mathbb{Z} .
$$

Under a lattice $\log$ concave assumption on the distribution of $M$, Parts 1 and 2 of Lemma 2.1 provide bounded couplings of random variables with distributions $\mathcal{L}(M \mid M \geq d)$ and $\mathcal{L}(M \mid M \leq$ d), respectively, to variables with distributions $\mathcal{L}(M \mid M \geq d+1)$ and $\mathcal{L}(M \mid M \leq d-1)$. Part 3 shows that there is a bounded coupling of $M$ to a variable having distribution $\mathcal{L}(M \mid M \neq d)$, provided $M$ is not degenerate at $d$. These results are extensions of [27], Lemma 3.3, that showed the $d=1$ case of Part 1 when $M$ is $\operatorname{Bin}(n, p)$ with $p \in(0,1)$.

In the following, we let $\operatorname{Bern}(p)$ denote the Bernoulli distribution giving mass $1-p$ and $p$ to 0 and 1 , respectively. 
Lemma 2.1. Let $M$ be a lattice $L C$ random variable with support $\mathcal{S} \subset \mathbb{Z}$.

1. For $x, d \in \mathbb{Z}$ define

$$
\pi_{x}^{(d)}= \begin{cases}\frac{P(M \geq x+1) P(M=d)}{P(M \geq d+1) P(M=x)}, & \text { if } x, d+1 \in \mathcal{S} \text { and } x \geq d, \\ 0, & \text { otherwise }\end{cases}
$$

Then the following hold.

(a) $0 \leq \pi_{x}^{(d)} \leq 1$ for all $x, d$.

(b) If $d+1 \in \mathcal{S}$ and $N, Z$ are random variables such that $\mathcal{L}(N)=\mathcal{L}(M \mid M \geq d)$ and $\mathcal{L}(Z \mid N)=\operatorname{Bern}\left(\pi_{N}^{(d)}\right)$, then $\mathcal{L}(N+Z)=\mathcal{L}(M \mid M \geq d+1)$.

2. For $x, d \in \mathbb{Z}$ define

$$
\rho_{x}^{(d)}= \begin{cases}\frac{P(M \leq x-1) P(M=d)}{P(M \leq d-1) P(M=x)}, & \text { if } x, d-1 \in \mathcal{S} \text { and } x \leq d, \\ 0, & \text { otherwise }\end{cases}
$$

Then the following hold.

(a) $0 \leq \rho_{x}^{(d)} \leq 1$ for all $x, d$.

(b) If $d-1 \in \mathcal{S}$ and $N, Z$ are random variables such that $\mathcal{L}(N)=\mathcal{L}(M \mid M \leq d)$ and $\mathcal{L}(Z \mid N)=\operatorname{Bern}\left(\rho_{N}^{(d)}\right)$, then $\mathcal{L}(N-Z)=\mathcal{L}(M \mid M \leq d-1)$.

3. Fix $d \in \mathbb{Z}$ such that $P(M=d)<1$. Let $Z_{+}, Z_{-}$be conditionally independent given $M$ with $\mathcal{L}\left(Z_{+} \mid M\right)=\operatorname{Bern}\left(\pi_{M}^{(d)}\right)$ and $\mathcal{L}\left(Z_{-} \mid M\right)=\operatorname{Bern}\left(\rho_{M}^{(d)}\right)$. Let $Z$ be independent of $Z_{+}$, $Z_{-}$, and $M$ with $\mathcal{L}(Z)=\operatorname{Bern}(q)$, where

$$
q=\frac{P(M \geq d+1)}{P(M \neq d)} .
$$

Then

$$
\mathcal{L}(M+X)=\mathcal{L}(M \mid M \neq d),
$$

where $X=Z Z_{+}-(1-Z) Z_{-}$.

In other words, the conclusion (16) says that, given $M$, a random variable with distribution $\mathcal{L}(M \mid M \neq d)$ can be formed by flipping an independent $q$-coin $Z$ and, if heads, adding 1 to $M$ with probability $\pi_{M}^{(d)}$, and otherwise subtracting 1 with probability $\rho_{M}^{(d)}$. We note that when $M<d$ (resp. $M>d$ ), the probability $\pi_{M}^{(d)}$ of adding (resp. $\rho_{M}^{(d)}$ of subtracting) 1 is 0 , and when $M=d, M$ is changed with probability 1 by either adding or subtracting 1 . We also note that when $M$ achieves the upper or lower limit of its support, the Bernoulli probability of adding to, or subtracting from $M$, respectively, is zero.

We define the hazard function of a lattice random variable $M$ with support $\mathcal{S}$ as

$$
h_{x}=\frac{P(M=x)}{P(M \geq x)}=\frac{p_{x}}{\sum_{y \geq x} p_{y}} \quad \text { for } x \in \mathcal{S} .
$$


To prove Lemma 2.1, we require the following fact that lattice LC distributions have nondecreasing hazard functions. This is well known for continuous LC distributions, for example, $[6,42]$.

Lemma 2.2. If $M$ is lattice $L C$ with support $\mathcal{S}$, then the hazard function $h_{x}$ given in (17) is nondecreasing on $\mathcal{S}$.

Proof. For any $x, y \in \mathcal{S}$ with $x \leq y$ note that by (15), we have

$$
\frac{p_{x+1}}{p_{x}} \geq \frac{p_{x+2}}{p_{x+1}} \geq \cdots \geq \frac{p_{y+1}}{p_{y}} .
$$

If $x, x+1 \in \mathcal{S}$, then

$$
\begin{aligned}
1 / h_{x}-1 / h_{x+1} & =\sum_{y \in \mathcal{S}: y \geq x} p_{y} / p_{x}-\sum_{y \in \mathcal{S}: y \geq x+1} p_{y} / p_{x+1}=\sum_{y \in \mathcal{S}: y \geq x}\left(p_{y} / p_{x}-p_{y+1} / p_{x+1}\right) \\
& =\sum_{y \in \mathcal{S}: y \geq x} \frac{p_{y}}{p_{x+1}}\left(\frac{p_{x+1}}{p_{x}}-\frac{p_{y+1}}{p_{y}}\right) \geq 0 .
\end{aligned}
$$

Proof of Lemma 2.1. Clearly $\pi_{x}^{(d)} \geq 0$, and to show that $\pi_{x}^{(d)} \leq 1$ it suffices to assume that $d, d+1 \in \mathcal{S}$ since $\pi_{x}^{(d)}=0$ otherwise. Let $h_{x}$ be the hazard function of $M$ defined by (17). For any $d \leq x \in \mathcal{S}$, by Lemma 2.2 we have $h_{d} \leq h_{x}$, and therefore

$$
\pi_{x}^{(d)}=\frac{1 / h_{x}-1}{1 / h_{d}-1} \leq 1
$$

proving Part 1a.

To prove Part 1b, letting $p_{x}=P(M=x)$ and $G_{x}=P(M \geq x)$, for any $k=1,2, \ldots$ we have

$$
\begin{aligned}
P(N+Z \geq d+k) & =P(N \geq d+k)+P(N=d+k-1, Z=1) \\
& =P(M \geq d+k \mid M \geq d)+\pi_{d+k-1}^{(d)} P(M=d+k-1 \mid M \geq d) \\
& =\frac{G_{d+k}}{G_{d}}+\left(\frac{G_{d+k} p_{d}}{G_{d+1} p_{d+k-1}}\right) \frac{p_{d+k-1}}{G_{d}}=\frac{G_{d+k}}{G_{d} G_{d+1}}\left(G_{d+1}+p_{d}\right) \\
& =\frac{G_{d+k}}{G_{d} G_{d+1}} G_{d}=\frac{G_{d+k}}{G_{d+1}}=P(M \geq d+k \mid M \geq d+1) .
\end{aligned}
$$

For Part 2a let $\tilde{M}=-M$, which is LC. For $d-1 \in \mathcal{S}$ and $d \geq x \in \mathcal{S}$,

$$
\rho_{x}^{(d)}=\frac{P(\tilde{M} \geq-x+1) P(\tilde{M}=-d)}{P(\widetilde{M} \geq-d+1) P(\widetilde{M}=-x)}=\widetilde{\pi}_{-x}^{(-d)} \in[0,1]
$$

by Part 1a, where $\tilde{\pi}$ is defined with respect to $\tilde{M}$. The rest of the proof of Part 2 is similar to that of Part 1. 
Moving to Part 3, letting $N$ denote the random variable $M+X$ on the LHS of (16), we will show that

$$
P(N \leq y)=P(M \leq y \mid M \neq d) \quad \text { for all } y \in \mathbb{Z}, y<d,
$$

the proof that $P(N \geq y)=P(M \geq y \mid M \neq d)$ for all $y>d$ being similar. Fix $y<d$ and without loss of generality assume that

$$
y+1 \in \mathcal{S}
$$

since otherwise (18) holds trivially as both sides are 0 or 1 . With $p_{x}, G_{x}$ as above and $F_{x}=$ $P(M \leq x)$,

$$
\begin{aligned}
P(N \leq y)= & P(M \leq y-1)+P(M=y, Z=0)+P\left(M=y, Z=1, Z_{+}=0\right) \\
& +P\left(M=y+1, Z=0, Z_{-}=1\right) \\
= & F_{y-1}+p_{y}(1-q)+p_{y} q\left(1-\pi_{y}^{(d)}\right)+p_{y+1}(1-q) \rho_{y+1}^{(d)} \\
= & F_{y}+p_{y+1}(1-q) \rho_{y+1}^{(d)},
\end{aligned}
$$

this last because $\pi_{y}^{(d)}=0$ since $y<d$. If $d-1 \in \mathcal{S}$, then (20) is

$$
\begin{aligned}
F_{y}+p_{y+1}\left(1-\frac{G_{d+1}}{1-p_{d}}\right)\left(\frac{F_{y} p_{d}}{F_{d-1} p_{y+1}}\right) & =F_{y}+\left(\frac{F_{d-1}}{1-p_{d}}\right) \frac{F_{y} p_{d}}{F_{d-1}} \\
& =\frac{F_{y}}{1-p_{d}}=P(M \leq y \mid M \neq d) .
\end{aligned}
$$

Otherwise $d-1 \notin \mathcal{S}$ so $\rho_{y+1}^{(d)}=0$, hence (20) is $F_{y}$. If $y=d-1$ then $\min \mathcal{S}=d$ by virtue of the assumption (19), so

$$
P(N \leq d-1)=F_{d-1}=0=P(M \leq d-1 \mid M \neq d) .
$$

In the remaining case, $d-1 \notin \mathcal{S}$ and $y \leq d-2$, we have $\max \mathcal{S}<d-1$ again by virtue of (19), and in particular $d \notin \mathcal{S}$. Then

$$
P(M \leq y \mid M \neq d)=P(M \leq y)=F_{y}=P(N \leq y),
$$

finishing the proof.

Corollary 2.1. Let $M$ be a lattice $L C$ random variable with support $\mathcal{S}$ satisfying a $:=\inf \mathcal{S}>$ $-\infty$, and let $d \in \mathcal{S}$. Then one can construct a random variable $A$ on the same space as $M$ such that $\mathcal{L}(M+A)=\mathcal{L}(M \mid M \geq d)$ and $0 \leq A \leq d-a$.

Proof. It suffices to prove the $a=0$ case because given $M$ satisfying the hypotheses and $d \in \mathcal{S}$ we have $d^{\prime}:=d-a \in \operatorname{supp}(M-a)$. Using that $M-a$ is an LC random variable, the $a=0$ case of the corollary guarantees $0 \leq A \leq d^{\prime}$ such that

$$
\mathcal{L}(M-a+A)=\mathcal{L}\left(M-a \mid M-a \geq d^{\prime}\right)=\mathcal{L}(M-a \mid M \geq d) .
$$


Using this fact, we have

$$
P(M+A \leq x)=P(M-a+A \leq x-a)=P(M-a \leq x-a \mid M \geq d)=P(M \leq x \mid M \geq d),
$$

which is the desired result.

To prove the $a=0$ case we successively construct random variables $M_{0}, \ldots, M_{d}$, all on the same space as $M$, such that

$$
\mathcal{L}\left(M_{k}\right)=\mathcal{L}(M \mid M \geq k) \quad \text { for } k=0,1, \ldots, d .
$$

Letting $M_{0}=M,(21)$ is satisfied for $k=0$. For $k=0, \ldots, d-1$, given $M_{0}, \ldots, M_{k}$ satisfying the distributional equality in (21), let $X_{k}$ be a Bernoulli random variable on the same space as $M_{0}, \ldots, M_{k}$ satisfying

$$
\mathcal{L}\left(X_{k} \mid M_{k}\right)=\operatorname{Bern}\left(\pi_{M_{k}}^{(k)}\right) \quad \text { and set } \quad M_{k+1}=M_{k}+X_{k} .
$$

It is easily checked that $\mathcal{L}(M)$ being lattice LC implies $\mathcal{L}\left(M_{k}\right)$ is LC. Hence Part $1 \mathrm{~b}$ of Lemma 2.1 yields that $M_{k+1}$ satisfies (21) for $k+1$. In particular, for $k=d$ our construction yields $M_{d}=M+A$ with $A=X_{0}+\cdots+X_{d-1}$ satisfying $0 \leq A \leq d$, and (21) yields the desired distributional property, concluding the proof.

Corollary 2.1, a consequence of Part 1 of Lemma 2.1, shows the existence of a 'uniformly close coupling' of an LC 'urn count' random variable $M$ to one with distribution $\mathcal{L}(M \mid M \geq d)$, and will be applied to coupling constructions for $Y_{\text {ge }}$. Similarly, Part 3 of Lemma 2.1, depending on Parts 1 and 2, will be applied to $Y_{\text {ne }}$.

Recall that a random variable $M$ is said to have a Poisson Binomial distribution with parameter $\mathbf{p}=\left(p_{j}\right)_{j \in[m]}$, denoted by $M \sim \mathcal{P B}(\mathbf{p})$, when

$$
\mathcal{L}(M)=\mathcal{L}\left(\sum_{j \in[m]} X_{j}\right),
$$

where $X_{j}$ are independent Bernoulli random variables with $P\left(X_{j}=1\right)=p_{j}$ for $j \in[m]$.

When there exists $p$ such that $p_{j}=p$ for all $j \in[m]$, then $M \sim \operatorname{Bin}(m, p)$. We note that the distribution of a single Bernoulli random variable, with support $\{0,1\}$, trivially satisfies (15) and hence is LC. Since [34] demonstrates that LC is preserved under convolution, the claim of the following lemma is immediate.

Lemma 2.3. The Poisson Binomial distribution $\mathcal{P B}(\mathbf{p})$ is $L C$.

When $M$ has distribution $\mathcal{P B}(\mathbf{p})$ for $\mathbf{p}=\left(p_{j}\right)_{j \in[m]}$, then for all $d \in \mathbb{Z}$ we have

$$
P(M=d)=q_{\mathrm{eq}}(d, \mathbf{p}) \quad \text { where } q_{\mathrm{eq}}(d, \mathbf{p})=\sum_{s \subset[m],|s|=d} \prod_{j \in s} p_{j} \prod_{j \notin s}\left(1-p_{j}\right)
$$

and so

$$
P(M \geq d)=q_{\mathrm{ge}}(d, \mathbf{p}) \quad \text { and } \quad P(M \neq d)=q_{\mathrm{ne}}(d, \mathbf{p}),
$$


where

$$
q_{\mathrm{ge}}(d, \mathbf{p})=\sum_{k=d}^{m} q_{\mathrm{eq}}(k, \mathbf{p}) \quad \text { and } \quad q_{\mathrm{ne}}(d, \mathbf{p})=1-q_{\mathrm{eq}}(d, \mathbf{p}) .
$$

With $\alpha \in[m]$, the majority of our constructions make use of the following definition for collections of counts of the form

$$
\left\{\mathbf{N}_{a}^{\alpha}=\left(N_{\beta, a}^{\alpha}\right)_{\beta \in[m]}: a \in \mathcal{S}_{\alpha}\right\}
$$

where $\mathcal{S}_{\alpha}, \alpha \in[m]$, are given support sets. Below, for given weight $\mathbf{w}=\left(w_{\alpha}\right)_{\alpha \in[m]}$ and threshold $\mathbf{d}=\left(d_{\alpha}\right)_{\alpha \in[m]}$ vectors, let

$$
|\mathbf{w}|=\max _{\alpha \in[m]} w_{\alpha} \quad \text { and } \quad|\mathbf{d}|=\max _{\alpha \in[m]} d_{\alpha} .
$$

Definition 2.2. For $B \geq 0$ and $\alpha \in[m]$, we say that the collection of counts (24) has Property $(B, \geq, \alpha)$ if

$$
\sum_{\beta \neq \alpha} w_{\beta} \mathbf{1}\left(N_{\beta, a+1}^{\alpha} \geq d_{\beta}\right) \leq \sum_{\beta \neq \alpha} w_{\beta} \mathbf{1}\left(N_{\beta, a}^{\alpha} \geq d_{\beta}\right)+|\mathbf{w}| B \quad \text { for all }\{a, a+1\} \subset \mathcal{S}_{\alpha},
$$

and Property $(B, \neq, \alpha)$ if

$$
\begin{aligned}
& \sum_{\beta \neq \alpha} w_{\beta} \mathbf{1}\left(N_{\beta, b}^{\alpha} \neq d_{\beta}\right) \leq \sum_{\beta \neq \alpha} w_{\beta} \mathbf{1}\left(N_{\beta, a}^{\alpha} \neq d_{\beta}\right)+|\mathbf{w}| B \\
& \quad \text { for all }\{a, b\} \subset \mathcal{S}_{\alpha} \text { with }|b-a|=1,
\end{aligned}
$$

for all $\mathbf{w}=\left(w_{1}, \ldots, w_{m}\right) \in(0, \infty)^{m}$ and all $\mathbf{d}=\left(d_{1}, \ldots, d_{m}\right) \in \mathbb{Z}^{m}$. If the counts have Property $(B, \geq, \alpha)$ (resp. $(B, \neq, \alpha))$ for all $\alpha \in[m]$, then they are said to have Property $(B, \geq)$ (resp. $(B, \neq))$.

For $\star \in\{\geq, \neq\}$ we say that a collection of configurations has Property $(B, \star)$ when their corresponding occupancy counts do.

The following claims are immediate.

1. For given $\alpha \in[m]$, counts (24) have Property $(B, \geq, \alpha)$ and $(B, \neq, \alpha)$ if

$$
\left|\left\{\beta: \beta \neq \alpha, N_{\beta, a}^{\alpha} \neq N_{\beta, a+1}^{\alpha}\right\}\right| \leq B \quad \text { whenever }\{a, a+1\} \in \mathcal{S}_{\alpha} .
$$

2. For given $\alpha \in[m]$, counts (24) have Property $(0, \geq, \alpha)$ when

$$
N_{\beta, a+1}^{\alpha} \leq N_{\beta, a}^{\alpha} \quad \text { for all }\{a, a+1\} \subset \mathcal{S}_{\alpha} \text { and } \beta \neq \alpha .
$$

3. For given $\alpha \in[m]$, if counts (24) have Property $(B, \geq, \alpha)$ then

$$
\sum_{\beta \neq \alpha} w_{\beta} \mathbf{1}\left(N_{\beta, b}^{\alpha} \geq d_{\beta}\right) \leq \sum_{\beta \neq \alpha} w_{\beta} \mathbf{1}\left(N_{\beta, a}^{\alpha} \geq d_{\beta}\right)+|\mathbf{w}| B(b-a)
$$

for all $\{a, b\} \subset \mathcal{S}_{\alpha}$ with $a \leq b$, all $\mathbf{w} \subset(0, \infty)^{m}$ and $\mathbf{d} \in \mathbb{Z}^{m}$. 
Now let $\mathcal{U}$ be a configuration corresponding to an occupancy model $\mathbf{M}=\left(M_{\alpha}\right)_{\alpha \in[m]}$, and for all $\alpha \in[m]$ let $\mathcal{S}_{\alpha}$ be the support of $M_{\alpha}, a_{\alpha}=\inf \mathcal{S}_{\alpha}$, and $b_{\alpha}=\sup \mathcal{S}_{\alpha}$. For $a \in \mathcal{S}_{\alpha}$ let

$$
\mathcal{L}\left(\mathcal{V}_{a}^{\alpha}\right):=\mathcal{L}\left(\mathcal{U} \mid M_{\alpha}=a\right)
$$

Theorem 2.1 is our main tool for the construction of bounded size biased couplings for $Y_{\mathrm{ge}}$ and $Y_{\text {ne }}$ for all applications other than those in Section 3.2. All constants $B$ in the following, whose value may change between different occurrences, are universal.

Theorem 2.1. Let $\mathcal{U}$ be a configuration corresponding to occupancy counts $\mathbf{M}=\left(M_{\alpha}\right)_{\alpha \in[m]}$, where for all $\alpha \in[\mathrm{m}]$ the component $M_{\alpha}$ is lattice $L C$. Suppose that for all $\alpha \in[m]$ there exists configurations $\left\{\mathcal{U}_{a}^{\alpha}, a \in \mathcal{S}_{\alpha}\right\}$ on a common space satisfying

$$
\mathcal{L}\left(\mathcal{U}_{a}^{\alpha}\right)=\mathcal{L}\left(\mathcal{V}_{a}^{\alpha}\right) \quad \text { for all } a \in \mathcal{S}_{\alpha},
$$

where $\mathcal{V}_{a}^{\alpha}$ is given by (31).

1. If $\left\{\mathcal{U}_{a}^{\alpha}, a \in \mathcal{S}_{\alpha}\right\}$ has Property $(B, \geq)$ and $a_{\alpha}>-\infty$ for all $\alpha \in[m]$, then there exists a coupling of variables $Y$ and $Y^{s}$ on the same space such that $\mathcal{L}(Y)=\mathcal{L}\left(Y_{\mathrm{ge}}\right)$ and $\mathcal{L}\left(Y^{s}\right)=$ $\mathcal{L}\left(Y_{\text {ge }}^{s}\right)$ satisfying

$$
Y^{s} \leq Y+|\mathbf{w}|(B|\mathbf{d}-\mathbf{a}|+1)
$$

where $\mathbf{d}-\mathbf{a}=\left(d_{\alpha}-a_{\alpha}\right)_{\alpha \in[m]}$.

2. If $\left\{\mathcal{U}_{a}^{\alpha}, a \in \mathcal{S}_{\alpha}\right\}$ has Property $(B, \neq)$ for all $\alpha \in[m]$, then there exists a coupling of variables $Y$ and $Y^{s}$ on the same space such that $\mathcal{L}(Y)=\mathcal{L}\left(Y_{\mathrm{ne}}\right)$ and $\mathcal{L}\left(Y^{s}\right)=\mathcal{L}\left(Y_{\mathrm{ne}}^{s}\right)$ satisfying

$$
Y^{s} \leq Y+|\mathbf{w}|(B+1) \text {. }
$$

Proof of Theorem 2.1. We prove (33) first. Fix $\alpha \in[m]$ and let $N_{\alpha}$ with distribution $\mathcal{L}\left(M_{\alpha}\right)$ be defined on the same space as, and independent of, the configurations $\left\{\mathcal{U}_{a}^{\gamma}, \gamma \in[m], a \in \mathcal{S}_{\gamma}\right\}$. By Corollary 2.1 one can construct $A_{\alpha}$ on the same space as $N_{\alpha}$ such that $\mathcal{L}\left(N_{\alpha}+A_{\alpha}\right)=$ $\mathcal{L}\left(M_{\alpha} \mid M_{\alpha} \geq d_{\alpha}\right)$ with $0 \leq A_{\alpha} \leq d_{\alpha}-a_{\alpha}$. In particular,

$$
\begin{aligned}
\mathcal{L}\left(\mathcal{U} \mid M_{\alpha} \geq d_{\alpha}\right) & =\sum_{a \geq d_{\alpha}, a \in \mathcal{S}_{\alpha}} \mathcal{L}\left(\mathcal{V}_{a}^{\alpha}\right) P\left(M_{\alpha}=a \mid M_{\alpha} \geq d_{\alpha}\right) \\
& =\sum_{a \geq d_{\alpha}, a \in \mathcal{S}_{\alpha}} \mathcal{L}\left(\mathcal{V}_{a}^{\alpha}\right) P\left(N_{\alpha}+A_{\alpha}=a\right)=\mathcal{L}\left(\mathcal{V}_{N_{\alpha}+A_{\alpha}}^{\alpha}\right)=\mathcal{L}\left(\mathcal{U}_{N_{\alpha}+A_{\alpha}}^{\alpha}\right)
\end{aligned}
$$

Clearly $\mathcal{L}\left(\mathcal{U}_{N_{\alpha}}^{\alpha}\right)=\mathcal{L}(\mathcal{U})$ by (31) and (32). Let $\mathbf{N}^{\alpha}$ and $\mathbf{N}_{\text {ge }}^{\alpha}$ be the counts corresponding to $\mathcal{U}_{N_{\alpha}}^{\alpha}$ and $\mathcal{U}_{N_{\alpha}+A_{\alpha}}^{\alpha}$, respectively. Since, for all $\alpha \in[m]$, the configurations $\left\{\mathcal{U}_{a}^{\alpha}, a \in \mathcal{S}_{\alpha}\right\}$ have Property $(B, \geq, \alpha)$ and $0 \leq A_{\alpha} \leq d_{\alpha}-a_{\alpha}$, we have

$$
\begin{aligned}
& \sum_{\beta \in[m]} w_{\beta} \mathbf{1}\left(N_{\beta, \mathrm{ge}}^{\alpha} \geq d_{\beta}\right)-\sum_{\beta \in[m]} w_{\beta} \mathbf{1}\left(N_{\beta}^{\alpha} \geq d_{\beta}\right) \\
& \quad \leq|\mathbf{w}|\left(B A_{\alpha}+1\right) \leq|\mathbf{w}|\left(B\left(d_{\alpha}-a_{\alpha}\right)+1\right) \leq|\mathbf{w}|(B|\mathbf{d}-\mathbf{a}|+1),
\end{aligned}
$$


where we applied observation (30), and where the factor +1 accounts for the maximum possible change from 0 to 1 of the indicator associated to urn $\alpha$.

Let $I$ be a random index with distribution (12) defined with respect to the weighted indicators in (3) summing to $Y_{\mathrm{ge}}$, independent of all other variables, and set $\mathbf{N}=\mathbf{N}^{I}$ and $\mathbf{N}_{\mathrm{ge}}=\mathbf{N}_{\mathrm{ge}}^{I}$. Now, for nonnegative integer counts $\mathbf{n}=\left(n_{\alpha}\right)_{\alpha \in[m]}$, setting

$$
Y(\mathbf{n})=\sum_{\alpha \in[m]} w_{\alpha} \mathbf{1}\left(n_{\alpha} \geq d_{\alpha}\right)
$$

averaging (36) over $\alpha$ distributed as $I$ we obtain

$$
Y\left(\mathbf{N}_{\mathrm{ge}}\right) \leq Y(\mathbf{N})+|\mathbf{w}|(B|\mathbf{d}-\mathbf{a}|+1) .
$$

The counts $\mathbf{N}$ have distribution $\mathcal{L}(\mathbf{M})$, as the same holds for $\mathbf{N}^{\alpha}$ for all $\alpha \in[m]$, by virtue of $\mathcal{L}\left(\mathcal{U}_{N_{\alpha}}^{\alpha}\right)=\mathcal{L}(\mathcal{U})$. In particular $Y=Y(\mathbf{N})$ has distribution $\mathcal{L}\left(Y_{\text {ge }}\right)$. By (35) the indicators $X_{\beta}^{\alpha}=$ $\mathbf{1}\left(N_{\beta, \text { ge }}^{\alpha^{\alpha}} \geq d_{\beta}\right)$ satisfy (10) with $X_{\beta}=\mathbf{1}\left(M_{\beta} \geq d_{\beta}\right)$, and Lemma 1.1 yields $Y^{s}=Y\left(\mathbf{N}_{\mathrm{ge}}\right)$ has the $Y_{\mathrm{ge}}$-size biased distribution. The proof of (33) is now complete.

To prove (34), first recall that we have reduced to the case that $P\left(M_{\alpha} \neq d_{\alpha}\right)<1$ for all $\alpha \in[m]$, allowing us to invoke Part 3 of Lemma 2.1. Construct $Z_{-}, Z_{+}$and $Z$ as in the lemma, on the same space as $M_{\alpha}$, so that with $A_{\alpha}=Z Z_{+}-(1-Z) Z_{-}$we have $\mathcal{L}\left(M_{\alpha}+A_{\alpha}\right)=\mathcal{L}\left(M_{\alpha} \mid M_{\alpha} \neq d_{\alpha}\right)$, with $-1 \leq A_{\alpha} \leq 1$. The proof proceeds in the same way as for (33).

Let $\mathbf{N}^{\alpha}$ and $\mathbf{N}_{\text {ne }}^{\alpha}$ be the counts corresponding to $\mathcal{U}_{N_{\alpha}}^{\alpha}$ and $\mathcal{U}_{N_{\alpha}+A_{\alpha}}^{\alpha}$, respectively. Since, for all $\alpha \in[m]$, the configurations $\left\{\mathcal{U}_{a}^{\alpha}, a \in \mathcal{S}_{\alpha}\right\}$ have Property $(B, \neq, \alpha)$ and $-1 \leq A_{\alpha} \leq 1$, we have

$$
\left|\sum_{\beta \in[m]} w_{\beta}\left(\mathbf{1}\left(N_{\beta, \text { ne }}^{\alpha} \neq d_{\beta}\right)-\mathbf{1}\left(N_{\beta}^{\alpha} \neq d_{\beta}\right)\right)\right| \leq|\mathbf{w}|\left(B\left|A_{\alpha}\right|+1\right) \leq|\mathbf{w}|(B+1) .
$$

Let $I$ be an independent index with distribution (12) defined with respect to the weighted indicators in (3) summing to $Y_{\text {ne }}$ and set $\mathbf{N}=\mathbf{N}^{I}$ and $\mathbf{N}_{\text {ne }}=\mathbf{N}_{\text {ne }}^{I}$. Then with $Y(\mathbf{n})$ given by (37) with $\geq$ replaced by $\neq$, we have $\mathcal{L}(Y(\mathbf{N}))=\mathcal{L}\left(Y_{\text {ne }}\right)$ and, by Lemma 1.1, that $\mathcal{L}\left(Y\left(\mathbf{N}_{\text {ne }}\right)\right)=\mathcal{L}\left(Y_{\text {ge }}^{s}\right)$. Now averaging (38) over $I$ yields

$$
\left|Y\left(\mathbf{N}_{\text {ne }}\right)-Y(\mathbf{N})\right| \leq|\mathbf{w}|(B+1),
$$

and the desired conclusion.

The following lemma is helpful in verifying that the conditions of Theorem 2.1 are in force when the configurations $\mathcal{U}$ corresponding to the occupancy counts $\mathbf{M}$ are given in terms of independent Bernoulli variables.

Lemma 2.4. Let $\mathcal{X}=\left(X_{\alpha}\right)_{\alpha \in[m]}$ be a collection of independent Bernoulli random variables with respective success probabilities $p_{1}, \ldots, p_{m} \in(0,1)$, and let $R=\sum_{\alpha \in[m]} X_{\alpha}$. Then there exists $\left\{\mathcal{X}_{a}, a \in[m]_{0}\right\}$ defined on a common space such that, for $a \in[m-1]_{0}$,

$$
\mathcal{L}\left(\mathcal{X}_{a}\right)=\mathcal{L}(\mathcal{X} \mid R=a) \quad \text { and } \quad \mathcal{X}_{a} \leq \mathcal{X}_{a+1} \quad \text { with probability one },
$$

where for $\{\mathbf{x}, \mathbf{y}\} \subset\{0,1\}^{m}$ we write $\mathbf{x} \leq \mathbf{y}$ when $x_{i} \leq y_{i}$ for all $i \in[m]$. 
Proof. Recall that the density $p(n)$ of an integer valued random variable is a Pólya frequency function of order 2 (or simply, is $\mathrm{PF}_{2}$; see [47]) when

$$
\left|\begin{array}{ll}
p\left(m_{1}-n_{1}\right) & p\left(m_{1}-n_{2}\right) \\
p\left(m_{2}-n_{1}\right) & p\left(m_{2}-n_{2}\right)
\end{array}\right| \geq 0 \quad \text { for all } m_{2} \geq m_{1} \text { and } n_{2} \geq n_{1} .
$$

For $\phi\left(y_{1}, \ldots, y_{m}\right)$ a coordinatewise non-decreasing function of $\left(y_{1}, \ldots, y_{m}\right) \in \mathbb{R}^{n}$, [19], Section 3, shows that if $Y_{1}, \ldots, Y_{m}$ are independent integer valued random variables with $\mathrm{PF}_{2}$ densities then $E\left(\phi\left(Y_{1}, \ldots, Y_{m}\right) \mid Y_{1}+\cdots+Y_{m}=a\right)$ is a non-decreasing function of $a$. As the Bernoulli density is $\mathrm{PF}_{2}$, we find in particular that

$$
E\left(\phi\left(X_{1}, \ldots, X_{m}\right) \mid R=a\right) \leq E\left(\phi\left(X_{1}, \ldots, X_{m}\right) \mid R=a+1\right) \quad \text { for all } a \in[m-1]_{0},
$$

for $\phi$ a coordinatewise non-decreasing function on $\{0,1\}^{m}$. See [10] for a simple proof of this fact in the Bernoulli case.

Relation (40) is expressed in Definition 2.1 of [38] as $\mathcal{X}_{a} \leq \mathcal{X}_{a+1}$ with probability one. Hence by Theorem 2.4 of [38] there exists a distribution $Q_{a}(\cdot, \cdot)$ on $\{0,1\}^{m} \times\{0,1\}^{m}$ for $(\mathcal{V}, \mathcal{W})$ such that

$$
\mathcal{L}(\mathcal{V})=\mathcal{L}\left(\mathcal{X}_{a}\right), \quad \mathcal{L}(\mathcal{W})=\mathcal{L}\left(\mathcal{X}_{a+1}\right) \quad \text { and } \quad \mathcal{V} \leq \mathcal{W} \quad \text { with probability one }
$$

(See [10] for a specific construction of the pair $(\mathcal{V}, \mathcal{W})$.)

With some slight abuse of notation, let $Q_{a}(\cdot \mid \cdot)$ denote the $Q_{a}(\cdot, \cdot)$ conditional distribution of the second argument given the first. Let $\mathcal{X}_{0}$ be the vector in $\{0,1\}^{m}$ with all coordinates equal to 0 , and for $a=1, \ldots, m$, given $\mathcal{X}_{a-1}$ let $\mathcal{X}_{a}$ be sampled from the conditional distribution $Q_{a}\left(\cdot \mid \mathcal{X}_{a-1}\right)$. Clearly $\mathcal{L}\left(\mathcal{X}_{a}\right)=\mathcal{L}(\mathcal{X} \mid R=a)$ for $a=0$. Assuming this identity holds for $a \in$ $[m-1]_{0}$, it holds also for $a+1$, as $\mathcal{L}\left(\mathcal{X}_{a+1}\right)$ is the conditional law $Q_{a+1}\left(\cdot \mid \mathcal{X}_{a}\right)$ averaged over the distribution $\mathcal{L}(\mathcal{X} \mid R=a)$, which equals $\mathcal{L}(\mathcal{X} \mid R=a+1)$ by construction.

Hence, the first property in (39) holds; the second is a consequence of the last relation in (41).

\section{Applications}

We now present in detail the three models mentioned in the Introduction, and use the constructions in Section 2 to prove concentration bounds for each case. With the exception of the volume of multi-way intersections in germ-grain models, the variables of interest are weighted occupancy counts of the form

$$
Y_{\mathrm{ge}}=\sum_{\alpha \in[m]} w_{\alpha} \mathbf{1}\left(M_{\alpha} \geq d_{\alpha}\right) \quad \text { and } \quad Y_{\mathrm{ne}}=\sum_{\alpha \in[m]} w_{\alpha} \mathbf{1}\left(M_{\alpha} \neq d_{\alpha}\right),
$$

although see the next paragraph for related random variables that can also be handled. Without loss of generality we assume that all summands in (42) are non-constant, for if a summand were constant then it could simply be subtracted from the corresponding $Y$ and the number of summands $m$ decremented by one. Some consequences of this assumption are that all $w_{\alpha}$ are 
strictly positive and, with $\mathcal{S}_{\alpha}$ denoting the support of $M_{\alpha}$, that inf $\mathcal{S}_{\alpha}<d_{\alpha}<\sup \mathcal{S}_{\alpha}+1$ for all $\alpha \in[m]$ when considering $Y_{\text {ge }}$, and $0<P\left(M_{\alpha} \neq d_{\alpha}\right)<1$ for all $\alpha \in[m]$ when considering $Y_{\text {ne }}$.

The concentration bounds we provide for variables of the form (42) also yield bounds for the 'complementary' sums

$$
\sum_{\alpha \in[m]} w_{\alpha} \mathbf{1}\left(M_{\alpha}<d_{\alpha}\right)=\sum_{\alpha \in[m]} w_{\alpha}-Y_{\mathrm{ge}} \quad \text { and } \sum_{\alpha \in[m]} w_{\alpha} \mathbf{1}\left(M_{\alpha}=d_{\alpha}\right)=\sum_{\alpha \in[m]} w_{\alpha}-Y_{\mathrm{ne}},
$$

with the mean $\mu=E Y$ replaced by $\sum_{\alpha \in[m]} w_{\alpha}-\mu$ and the roles of the right and left tails reversed. In fact, all our results can be extended further, with essentially only a notational burden, to random variables of the form

$$
Y=\sum_{\alpha \in[m]} w_{\alpha} \mathbf{1}\left(M_{\alpha} \star_{\alpha} d_{\alpha}\right) \quad \text { where } \star_{\alpha} \in\{\geq, \neq\},
$$

and therefore, in like manner, to the sums of complementary form.

Lastly, we note that when $M_{\alpha} \sim \mathcal{P B}\left(\mathbf{p}_{\alpha}\right)$ for each $\alpha \in[m]$, by (23) the means $\mu_{\mathrm{ge}}$ and $\mu_{\mathrm{ne}}$ of $Y_{\mathrm{ge}}$ and $Y_{\mathrm{ne}}$ are given respectively, by

$$
\mu_{\mathrm{ge}}=\sum_{\alpha \in[m]} w_{\alpha} q_{\mathrm{ge}}\left(d_{\alpha}, \mathbf{p}_{\alpha}\right) \quad \text { and } \quad \mu_{\mathrm{ne}}=\sum_{\alpha \in[m]} w_{\alpha} q_{\mathrm{ne}}\left(d_{\alpha}, \mathbf{p}_{\alpha}\right),
$$

where $q_{\mathrm{ge}}$ and $q_{\mathrm{ne}}$ are given by (23).

\subsection{Degree counts in Erdôs-Rényi type graphs}

The classical Erdős-Rényi random graph on $m$ vertices is constructed by placing an edge between each pair of distinct vertices independently and with equal probability. The model was originally used in conjunction with the probabilistic method for proving the existence of graphs with certain properties (see [1]) and has been popular more recently for modeling complex networks (e.g., [14]).

The classical Erdős-Rényi graph with constant connectivity $p$ has been the object of much study. Asymptotic normality of the number of vertices of degree $d$ was shown in [33] when $m^{(d+1) / d} \rightarrow \infty$ and $m p \rightarrow 0$, or $m p \rightarrow 0$ and $m p-\log m-d \log \log m \rightarrow-\infty$. Asymptotic normality when $m p \rightarrow c>0$ was obtained in [7]. Optimal bounds in the Kolmogorov metric can be found in [36] and [26]. Other univariate results on asymptotic normality of counts on random graphs are given in [31], and references therein. Smooth function bounds were obtained in [28] for the vector whose $k$ components count the number of vertices of fixed degrees $d_{1}, d_{2}, \ldots, d_{k}$ when $p=\theta /(m-1) \in(0,1)$ for fixed $\theta$, implying asymptotic multivariate joint normality. This work was later extended in [39] to the inhomogeneous random graph model which will be the setting in the current paper.

Here we consider graph degree counts when the likelihood of an edge may depend on the identity of the vertices it connects. Formally, let $\mathcal{G}_{m}$ be an Erdős-Rényi random graph on the vertices $[m]$, where the presence of an edge joining distinct vertices $\alpha$ and $\beta$ is recorded by the 
indicator $X_{\{\alpha, \beta\}}$ with success probability $p_{\{\alpha, \beta\}}$, with all such indicators independent. We set $p_{\{\alpha, \alpha\}}=0$ for all $\alpha \in[m]$, making all $X_{\alpha, \alpha}$ identically zero. The classical model is recovered by setting $p_{\{\alpha, \beta\}}=p$ for some $p \in[0,1]$ for all $\alpha \neq \beta$.

For $Y_{\mathrm{ge}}$, with similar remarks applying to $Y_{\mathrm{ne}}$, by removing any edge $\{\alpha, \beta\}$ with $p_{\{\alpha, \beta\}}=1$ and decrementing each of the two thresholds $d_{\alpha}, d_{\beta}$ by one we may assume that $p_{\{\alpha, \beta\}}<1$ for all $(\alpha, \beta) \in[m] \times[m]$. Having also reduced to the case where all the indicators in (42) are nontrivial allows us to assume that $\sum_{\beta: \beta \neq \alpha} p_{\{\alpha, \beta\}}>0$ for all $\alpha \in[m]$.

Let the components $M_{\alpha}$ of $\mathbf{M}=\left(M_{\alpha}\right)_{\alpha \in[m]}$ record the degree of vertex $\alpha$, that is, $M_{\alpha}=$ $\sum_{\beta \in[m]} X_{\{\alpha, \beta\}}$. By the definition (22), $M_{\alpha} \sim \mathcal{P B}\left(\mathbf{p}_{\alpha}\right)$ with $\mathbf{p}_{\alpha}=\left(p_{\{\alpha, \beta\}}\right)_{\beta: \beta \neq \alpha}$. By (23) the means $\mu_{\mathrm{ge}}$ and $\mu_{\mathrm{ne}}$ of $Y_{\mathrm{ge}}$ and $Y_{\mathrm{ne}}$ have the form (43). With $|\mathbf{w}|$ and $|\mathbf{d}|$ as in (25), let

$$
c_{\mathrm{ge}}=|\mathbf{w}|(|\mathbf{d}|+1) \quad \text { and } \quad c_{\mathrm{ne}}=2|\mathbf{w}| .
$$

Theorem 3.1. Concentration of measure inequalities (5)-(8) hold for counts $Y_{\mathrm{ge}}$ and $Y_{\mathrm{ne}}$ given by (42) in $\mathcal{G}_{m}$ for all $m \geq 1$, with corresponding $\mu$ and $c$ given by (43) and (44).

Theorem 3.1 is a direct consequence of the following lemma.

Lemma 3.1. In the $\mathcal{G}_{m}$ model there exists a coupling of $Y_{\mathrm{ge}}$ to $Y_{\mathrm{ge}}^{s}$, having the $Y_{\mathrm{ge}}$-size biased distribution, that satisfies $Y_{\mathrm{ge}}^{s}-Y_{\mathrm{ge}} \leq c_{\mathrm{ge}}$, and a coupling of $Y_{\mathrm{ne}}$ to $Y_{\mathrm{ne}}^{s}$, having the $Y_{\mathrm{ne}}$-size biased distribution, satisfying $Y_{\mathrm{ne}}^{s}-Y_{\mathrm{ne}} \leq c_{\mathrm{ne}}$.

Proof. In this model, we take the configuration

$$
\mathcal{U}=\left\{X_{\{\gamma, \delta\}},\{\gamma, \delta\} \subset[m]\right\},
$$

the collection of the independent Bernoulli edge indicator variables of the graph $\mathcal{G}_{m}$. As the corresponding counts $\mathbf{M}$ have LC marginal distributions with supports $\mathcal{S}_{\alpha}$ satisfying inf $\mathcal{S}_{\alpha}=0$, in order to invoke Theorem 2.1 it is only required to show that for all $\alpha \in[m]$, configurations $\left\{\mathcal{U}_{a}^{\alpha}, a \in[m]\right\}$ exist with Properties $(1, \geq)$ and $(1, \neq)$ satisfying $(32)$.

With $a \in \mathcal{S}_{\alpha}$ and $\mathcal{V}_{a}^{\alpha}$ as in (31), by independence we obtain

$$
\begin{aligned}
\mathcal{L}\left(\mathcal{V}_{a}^{\alpha}\right) & =\mathcal{L}\left(\mathcal{U} \mid M_{\alpha}=a\right)=\mathcal{L}\left(X_{\{\gamma, \delta\}},\{\gamma, \delta\} \subset[m] \mid M_{\alpha}=a\right) \\
& =\mathcal{L}\left(X_{\{\gamma, \delta\}},\{\gamma, \delta\} \subset[m] \mid \sum_{\delta \in[m]} X_{\{\alpha, \delta\}}=a\right) \\
& =\mathcal{L}\left(X_{\{\alpha, \delta\}}, \delta \in[m] \mid \sum_{\delta \in[m]} X_{\{\alpha, \delta\}}=a\right) \times \mathcal{L}\left(X_{\{\gamma, \delta\}},\{\gamma, \delta\} \not \supset \alpha\right),
\end{aligned}
$$

where here $x$ denotes product measure.

On the same space and independently of $\mathcal{U}$ and of each other over $\alpha \in[\mathrm{m}]$, let

$$
\mathcal{X}_{a}^{\alpha}=\left\{X_{\{\alpha, \delta\}, a}^{\alpha}, \delta \in[m]\right\}, \quad a \in[m]
$$


be the collections of Bernoulli variables guaranteed by Lemma 2.4 when taking $\left(X_{\alpha}\right)_{\alpha \in[m]}$ in the lemma to be $\left(X_{\{\alpha, \delta\}}\right)_{\delta \in[m]}$. In particular

$$
\mathcal{L}\left(X_{\{\alpha, \delta\}, a}^{\alpha}, \delta \in[m]\right)=\mathcal{L}\left(X_{\{\alpha, \delta\}}, \delta \in[m] \mid \sum_{\delta \in[m]} X_{\{\alpha, \delta\}}=a\right),
$$

and setting $\mathcal{U}_{a}^{\alpha}=\left\{X_{\{\alpha, \delta\}, a}^{\alpha}, \delta \in[m], X_{\left\{\gamma, \delta^{\prime}\right\}},\left\{\gamma, \delta^{\prime}\right\} \not \supset \alpha\right\}$, we obtain $\mathcal{L}\left(\mathcal{U}_{a}^{\alpha}\right)=\mathcal{L}\left(\mathcal{V}_{a}^{\alpha}\right)$.

Again by Lemma 2.4, with $a+1 \in \mathcal{S}_{\alpha}$, the Bernoulli variables equal to one in the collection $\mathcal{X}_{a+1}^{\alpha}$ are those equal to one in $\mathcal{X}_{a}^{\alpha}$, with one additional variable. The configurations $\mathcal{U}_{a}^{\alpha}$ and $\mathcal{U}_{a+1}^{\alpha}$ therefore correspond to graphs $\mathcal{G}_{m, a}^{\alpha}$ and $\mathcal{G}_{m, a+1}^{\alpha}$, where the edge set of the latter is that of the former plus exactly one additional edge attached to vertex $\alpha$. In particular, the corresponding counts $\mathbf{N}_{a}^{\alpha}$ and $\mathbf{N}_{a+1}^{\alpha}$ agree in all but coordinate $\alpha$ and one additional coordinate. Hence, (28) is satisfied with $B=1$, implying that the configurations $\left\{\mathcal{U}_{a}^{\alpha}, a \in[m]\right\}$ have both Properties $(1, \geq)$ and $(1, \neq)$, thus completing the proof.

In the standard case of equal thresholds $d_{\alpha}=d$ and unit weightings, the expectations (43) of $Y_{\text {ge }}$ and $Y_{\text {ne }}$ simplify to

$$
\mu_{\mathrm{ge}}=m P(\operatorname{Bin}(m-1, p) \geq d) \quad \text { and } \quad \mu_{\mathrm{ne}}=m P(\operatorname{Bin}(m-1, p) \neq d),
$$

respectively, and the bounds (5)-(8) apply to $Y_{\text {ge }}$ with $c=d+1$, and for $Y_{\text {ne }}$ with $c=2$. In particular, (5) and (8) yield that, for all $t>0$,

$$
\begin{gathered}
P\left(Y_{\mathrm{ge}}-\mu_{\mathrm{ge}} \leq-t\right) \leq \exp \left(-\frac{t^{2}}{2(d+1) \mu_{\mathrm{ge}}}\right) \text { and } \\
P\left(Y_{\mathrm{ge}}-\mu_{\mathrm{ge}} \geq t\right) \leq \exp \left(-\frac{t^{2}}{2(d+1)\left(\mu_{\mathrm{ge}}+t / 3\right)}\right) .
\end{gathered}
$$

The special case of the number of isolated vertices

$$
Y_{\text {is }}=\sum_{\alpha \in[m]} \mathbf{1}\left(M_{\alpha}=0\right)
$$

for the standard Erdős-Rényi model was handled in [25], using an unbounded size bias coupling, and with much greater effort. Techniques of the present paper can be used to obtain concentration bounds for $Y_{\text {is }}$ in a much simpler way by noting that $m-Y_{\text {is }}=Y_{\text {ge }}$ under unit weightings and equal thresholds $d_{\alpha}=1$. In particular, the bounds (46) hold with $Y_{\mathrm{is}}-\mu_{\text {is }}$ replacing $Y_{\mathrm{ge}}-\mu_{\mathrm{ge}}$ and setting $d=1$, reversing the roles of the left and right tail bounds, and replacing $\mu_{\mathrm{ge}}$ by $m-\mu_{\text {is }}$. The left tail bound obtained in this fashion is stronger than the corresponding bound

$$
P\left(Y_{\text {is }}-\mu_{\text {is }} \leq-t\right) \leq \exp \left(-\frac{t^{2}}{4 \mu_{\text {is }}}\right)
$$

given in [25], for $t \leq 6 m(1-p)^{m-1}-3 m$, with similar remarks applying to the right tail. 
Although the unbounded size bias coupling argument given in [25] applies only to the case of isolated vertices, Theorem 3.1 applies equally for all degrees $d$. In particular, keeping $p$ and $d$ fixed and letting $m \rightarrow \infty$, the left and right tail bounds for $Y_{\text {ge }}$ provided by (5) and (8), say, will behave as $\exp \left(-t^{2} /(2(d+1) m)\right)$ and $\exp \left(-t^{2} /(2(d+1)(m+t / 3))\right)$, respectively.

A well studied asymptotic is the case where $d$ is fixed and $m p \rightarrow \lambda$ for some $\lambda>0$ so that for large $m$, the distribution $\operatorname{Bin}(m-1, p)$ is close to a Poisson random variable with parameter $\lambda$. Here focusing on the statistic $Y_{\mathrm{ge}}=\sum_{\alpha \in[m]} \mathbf{1}\left(M_{\alpha} \geq 1\right)$ for simplicity, the mean satisfies $\mu_{\mathrm{ge}} \rightarrow$ $m\left(1-e^{-\lambda}\right)$, and the resulting left and right tail bounds are asymptotic to $\exp \left(-t^{2} /\left(4 m\left(1-e^{-\lambda}\right)\right)\right)$ and $\exp \left(-t^{2} /\left(4 m\left(1-e^{-\lambda}\right)+4 t / 3\right)\right)$, respectively, as $m \rightarrow \infty$. Comparisons of these tail bounds with other techniques from the literature will be discussed in detail in Section 4.

\subsection{Germ-grain models}

Germ-grain models consist of sets (grains) placed at centers (germs) determined by a random point process in some multidimensional space. These models are used in applications including forestry [49], material science [2] and wireless sensor networks [17]. For concreteness, here we consider models on the space $C_{n}=\left[0, n^{1 / p}\right)^{p} \subset \mathbb{R}^{p}$, equipped with the Euclidean toroidal distance $D$.

In the models considered in this section, a configuration $\mathcal{V}$ is given by a collection $\left(v_{\alpha}\right)_{\alpha \in[m]}$ of points in $C_{n}$, and each point $v_{\alpha}$ is associated with a closed ball $B_{\alpha}$ centered at $v_{\alpha}$ with positive radius $\rho_{\alpha}$. We let $\mathcal{U}$ consist of points $U_{1}, \ldots, U_{m}$ sampled independently in $C_{n}$ with strictly positive densities $f_{1}(x), \ldots, f_{m}(x)$ in $C_{n}$, respectively. The positivity condition on the densities are assumed for convenience, as along with (48) in Section 3.2.1, and (62) in Section 3.2.2, respectively, it implies that the support is $[m]_{0}$ for the number $M(x, \mathcal{U})$ of intersections of $\mathcal{U}$ at a point $x \in C_{n}$, defined below in (47), and support [ $\left.m-1\right]_{0}$ for the number $M_{\alpha}$ of neighbors of $U_{\alpha}, \alpha \in[m]$, defined below in (61).

\subsubsection{Volume of multi-way intersections in germ-grain models}

For a point $x \in C_{n}$ and a configuration $\mathcal{V}$, let

$$
M(x, \mathcal{V})=\sum_{\alpha \in[m]} \mathbf{1}\left(x \in B_{\alpha}\right),
$$

the number of balls $B_{\alpha}$ that contain the point $x$ in the configuration $\mathcal{V}$. In this subsection, we make the assumption that $n$ is large enough that

$$
\sqrt{p} n^{1 / p}>2 \sum_{\alpha \in[m]} \rho_{\alpha} .
$$

Under (48) there exist $\left(v_{\alpha}\right)_{\alpha \in[m]} \subset C_{n}$ such that $B_{\alpha} \cap B_{\beta}=\varnothing$ for all $\alpha \neq \beta$, implying the support of $M(x, \mathcal{U})$ is $[m]_{0}$. 
For a fixed measurable function $d: C_{n} \rightarrow[m]$ and $f \in[m]$ let

$$
\mathbf{1}_{x, \mathrm{ge}}(\mathcal{V})=\mathbf{1}\left(x \in B_{\mathrm{ge}}(d(x), \mathcal{V})\right) \quad \text { where } B_{\mathrm{ge}}(f, \mathcal{V})=\bigcup_{\substack{r \subset[m] \\|r| \geq f}} \bigcap_{\alpha \in r} B_{\alpha} .
$$

Hence, dropping the dependence on $\mathcal{V}$ unless clarity demands it, $\mathbf{1}_{x, \mathrm{ge}}=1$ if and only if $x$ is contained in at least $d(x)$ of the balls $B_{\alpha}, \alpha \in[m]$. Further, emphasizing dependence on the function $d$ by writing $\mathbf{1}_{x, d}=\mathbf{1}_{x, \text { ge }}$, the indicators

$$
\mathbf{1}_{x, \mathrm{eq}}=\mathbf{1}_{x, d}-\mathbf{1}_{x, d+1} \quad \text { and } \quad \mathbf{1}_{x, \mathrm{ne}}=1-\mathbf{1}_{x, \mathrm{eq}}
$$

take the value 1 if $x$ is, and is not, contained in exactly $d(x)$ of the balls $B_{\alpha}, \alpha \in[m]$, respectively. In particular, given a nonnegative, bounded function $w(x)$ over $C_{n}$,

$$
Y_{\mathrm{ge}}(\mathcal{V})=\int_{C_{n}} w(x) \mathbf{1}_{x, \mathrm{ge}}(\mathcal{V}) d x \quad \text { and } \quad Y_{\mathrm{ne}}(\mathcal{V})=\int_{C_{n}} w(x) \mathbf{1}_{x, \mathrm{ne}}(\mathcal{V}) d x
$$

are the volumes, weighted by $w(x)$, of the collection of points $x$ in $C_{n}$ contained in at least $d(x)$ balls, and some number of balls other than $d(x)$, respectively. When $w(x)=1$ the variables in (50) are the volumes of the sets of points $x \in C_{n}$ that are part of $d(x)$ way intersections, and intersections of size other than $d(x)$, respectively.

Letting

$$
p_{\alpha}(x)=P\left(D\left(x, U_{\alpha}\right) \leq \rho_{\alpha}\right), \quad \alpha \in[m],
$$

the variable $M(x, \mathcal{U})$ of (47) has the Poisson Binomial distribution $\mathcal{P} \mathcal{B}(\mathbf{p}(x))$. As, for instance, $\left\{x \in B_{\text {ge }}(x, \mathcal{V})\right\}=\{M(x, \mathcal{V}) \geq d(x)\}$, we may write

$$
\begin{aligned}
& Y_{\mathrm{ge}}(\mathcal{V})=\int_{C_{n}} w(x) \mathbf{1}(M(x, \mathcal{V}) \geq d(x)) d x \quad \text { and } \\
& Y_{\mathrm{ne}}(\mathcal{V})=\int_{C_{n}} w(x) \mathbf{1}(M(x, \mathcal{V}) \neq d(x)) d x
\end{aligned}
$$

whose expectations when $\mathcal{V}=\mathcal{U}$ are given respectively by

$$
\mu_{\mathrm{ge}}=\int_{C_{n}} w(x) q_{\mathrm{ge}}(d(x), \mathbf{p}(x)) d x \quad \text { and } \quad \mu_{\mathrm{ne}}=\int_{C_{n}} w(x) q_{\mathrm{ne}}(d(x), \mathbf{p}(x)) d x .
$$

The size biased couplings for the germ-grain models in this subsection and the following one are simple extensions of those in Section 4 of [27]. There are three differences between the present case and that of [27]. First, in that previous work one considers only $d(x)=1$ for all $x \in$ $C_{n}$ for the covered volume. Next, $U_{1}, \ldots, U_{m}$ were taken in [27] to have the uniform distribution over $C_{n}$, and lastly the radii $\rho_{\alpha}$ were set to some fixed $\rho$ for all $\alpha \in[m]$. The imposition of these conditions result in various simplifications in [27], which the following outline generalizes in these aspects. 
Let $U_{0}$ be sampled independently of $\mathcal{U}$ with density $f_{0}(x)=w(x) \mathbf{1}\left(x \in C_{n}\right) / w$ where $w=$ $\int_{C_{n}} w(x) d x$, and for $u \in C_{n}$ consider the event $F(u)=\{M(u, \mathcal{U}) \geq d(u)\}$ that the point $u$ lies in a $d(u)$ way intersection. Then, in view of (52), it is easy to see that $Y_{\text {ge }}(\mathcal{U})=w P\left(F\left(U_{0}\right) \mid \mathcal{F}\right)$ where $\mathcal{F}$ is the $\sigma$-algebra generated by $\left\{U_{\alpha}, \alpha \in[m]\right\}$. Hence, by (9), $E\left(Y_{\text {ge }}(\mathcal{U}) \mid F\left(U_{0}\right)\right)$ has the $Y_{\text {ge }}(\mathcal{U})$ size biased distribution. In particular, letting $Y_{\text {ge }}^{S}$ denote a variable with the $Y_{\text {ge }}(\mathcal{U})$-size biased distribution, we have that

$$
\mathcal{L}\left(Y_{\text {ge }}\left(\mathcal{U}^{0}\right)\right)=\mathcal{L}\left(Y_{\text {ge }}^{s}\right) \quad \text { where } \mathcal{L}\left(\mathcal{U}^{0}\right)=\mathcal{L}\left(\mathcal{U} \mid F\left(U_{0}\right)\right)
$$

that is, the law of $\mathcal{U}^{0}$ is that of the configuration $\mathcal{U}$ conditioned on the event that the additional randomly chosen point $U_{0}$ lies in a $d\left(U_{0}\right)$ way intersection.

In the following, for $\alpha \in[m], u \in C_{n}$ and $i \in\{0,1\}$ let $f_{\alpha, u, i}$ be the distributions specified by

$$
\begin{aligned}
& f_{\alpha, u, 0}(E)=P\left(U_{\alpha} \in E \mid D\left(U_{\alpha}, u\right)>\rho_{\alpha}\right) \quad \text { and } \\
& f_{\alpha, u, 1}(E)=P\left(U_{\alpha} \in E \mid D\left(U_{\alpha}, u\right) \leq \rho_{\alpha}\right) .
\end{aligned}
$$

With the given weight, threshold functions $w(x), d(x)$ and radii $\left(\rho_{\alpha}\right)_{\alpha \in[m]}$, let

$$
|\mathbf{w}|=\sup _{x \in C_{n}}|w(x)|, \quad|\mathbf{d}|=\sup _{x \in C_{n}}|d(x)| \quad \text { and } \quad|\boldsymbol{\rho}|=\max _{\alpha \in[m]} \rho_{\alpha},
$$

and with $\pi_{p}$ the volume of the unit ball in $\mathbb{R}^{p}$, let

$$
c_{\mathrm{ge}}=\pi_{p}|\mathbf{w}||\mathbf{d}||\rho|^{p} \quad \text { and } \quad c_{\mathrm{ne}}=\pi_{p}|\mathbf{w}||\rho|^{p} .
$$

Theorem 3.2. Concentration of measure inequalities (5)-(8) hold for all $m \geq 1$ for the volume covered by multi-way intersections in the germ-grain model described above, with $Y, \mu$ and $c$ given by (50), (53) and (56).

Again, Theorem 3.2 follows from the following bounded coupling construction.

Lemma 3.2. In the germ-grain model described above there exists a coupling of $Y_{\mathrm{ge}}$ to $Y_{\mathrm{ge}}^{s}$, having the $Y_{\mathrm{ge}}$-size biased distribution, that satisfies $Y_{\mathrm{ge}}^{s} \leq Y_{\mathrm{ge}}+c_{\mathrm{ge}}$, and a coupling of $Y_{\mathrm{ne}}$ to $Y_{\mathrm{ne}}^{s}$, having the $Y_{\mathrm{ne}}$-size biased distribution, satisfying $Y_{\mathrm{ne}}^{s} \leq Y_{\mathrm{ne}}+c_{\mathrm{ne}}$.

Proof. As for any event $E$ we have

$$
P\left(E \mid F\left(U_{0}\right)\right)=\frac{P\left(E, F\left(U_{0}\right)\right)}{P\left(F\left(U_{0}\right)\right)}=\int_{C_{n}} \frac{P(E, F(u))}{P(F(u))} \frac{P(F(u))}{P\left(F\left(U_{0}\right)\right)} \frac{w(u)}{w} d u,
$$

we see $\mathcal{L}\left(\mathcal{U} \mid F\left(U_{0}\right)\right)$ of (54) is the mixture

$$
\mathcal{L}\left(\mathcal{U} \mid F\left(U_{0}\right)\right)=\int_{C_{n}} \mathcal{L}(\mathcal{U} \mid F(u)) \widetilde{w}(u) d u \quad \text { where } \widetilde{w}(u)=\frac{P(F(u))}{P\left(F\left(U_{0}\right)\right)} \frac{w(u)}{w} .
$$


Hence, we sample $\widetilde{U}$ on $C_{n}$ with density $\widetilde{w}(u)$, and for $\widetilde{U}=u$, construct a configuration $\mathcal{U}$ with law $\mathcal{L}(\mathcal{U} \mid F(u))$ in order to achieve $\mathcal{L}\left(\mathcal{U} \mid F\left(U_{0}\right)\right)$; see Lemma 2.3 of [4] regarding size biasing mixture distributions.

For $u \in C_{n}$ let $\left(X_{\alpha, u}\right)_{\alpha \in[m]}$ be independent Bernoulli variables with success probabilities $\left(p_{\alpha}(u)\right)_{\alpha \in[m]}$ as given in (51), and for $a \in[m]_{0}$, let

$$
P\left(X_{1, u, a}=i_{1}, \ldots, X_{m, u, a}=i_{m}\right)=P\left(X_{1, u}=i_{1}, \ldots, X_{m, u}=i_{m} \mid \sum_{\alpha \in[m]} i_{\alpha}=a\right)
$$

and

$$
\mathcal{X}_{a}=\left\{X_{\alpha, u, a}, \alpha \in[m]\right\}, \quad a \in[m]_{0}
$$

be the collection of Bernoulli variables guaranteed by Lemma 2.4 for the vector of success probabilities $\left(p_{\alpha}(u)\right)_{\alpha \in[m]}$.

For $\alpha \in[m]$ let $U_{\alpha ; 0}$ and $U_{\alpha ; 1}$ have distributions $f_{\alpha, u, 0}$ and $f_{\alpha, u, 1}$ respectively, as specified in (55). Then for $a \in[m]_{0}$, writing $U_{\alpha, a}=U_{\alpha ; X_{\alpha, u, a}}$ and letting $B_{\alpha, a}$ be the ball of radius $\rho_{\alpha}$ centered at $U_{\alpha, a}$, the configuration $\mathcal{U}_{a}=\left\{U_{\alpha, a}, \alpha \in[m]\right\}$ satisfies $\mathcal{L}\left(\mathcal{U}_{a}\right)=\mathcal{L}(\mathcal{U} \mid M(u, \mathcal{U})=a)$.

Let $N_{u}$ be constructed on the same space with distribution $\mathcal{L}(M(u, \mathcal{U}))$. Applying Corollary 2.1 to $M(u, \mathcal{U})$, we obtain $A_{u}$ satisfying

$$
\mathcal{L}\left(N_{u}+A_{u}\right)=\mathcal{L}(M(u, \mathcal{U}) \mid F(u)) \quad \text { with } 0 \leq A_{u} \leq d(u) .
$$

Denoting the underlying configurations corresponding to $N_{u}$ and to $N_{u}+A_{u}$, by $\mathcal{U}_{N_{u}}$ and $\mathcal{U}_{N_{u}+A_{u}}$, respectively, we have $\mathcal{L}\left(\mathcal{U}_{N_{u}+A_{u}}\right)=\mathcal{L}(\mathcal{U} \mid F(u))$. Since $\tilde{U}=u$ was sampled according to density $\widetilde{w}(u)$, by (57) for the second identity, letting $N=N_{\tilde{U}}$ and $A=A_{\tilde{U}}$,

$$
\mathcal{L}\left(\mathcal{U}_{N}\right)=\mathcal{L}(\mathcal{U}) \quad \text { and } \quad \mathcal{L}\left(\mathcal{U}_{N+A}\right)=\mathcal{L}\left(\mathcal{U} \mid F\left(U_{0}\right)\right)
$$

By (54), $Y_{\text {ge }}^{s}=Y_{\text {ge }}\left(\mathcal{U}_{N+A}\right)$ has the $Y_{\mathrm{ge}}=Y\left(\mathcal{U}_{N}\right)$ size biased distribution.

Note that (39) of Lemma 2.4 implies that for $a \in[m-1]_{0}$ the indicators $\mathcal{X}_{a+1}$ and $\mathcal{X}_{a}$ are equal, but for one index, say $\beta_{a}$, such that $X_{\beta_{a}, U_{0}, a}=0$ and $X_{\beta_{a}, U_{0}, a+1}=1$. Hence, the configurations $\mathcal{U}_{a}$ and $\mathcal{U}_{a+1}$ are the same but for the one point indexed by $\beta_{a}$. Therefore, with $B_{\mathrm{ge}, a}$ given by (49) with $B_{\alpha}$ replaced by $B_{\alpha, a}$,

$$
\mathbf{1}_{x, \text { ge }}\left(\mathcal{U}_{a+1}\right) \leq \mathbf{1}_{x, \text { ge }}\left(\mathcal{U}_{a}\right) \mathbf{1}\left(x \notin B_{\beta_{a}}^{a+1}\right)+\mathbf{1}\left(x \in B_{\beta_{a}}^{a+1}\right) \leq \mathbf{1}_{x, \mathrm{ge}}\left(\mathcal{U}_{a}\right)+\mathbf{1}\left(x \in B_{\beta_{a}}^{a+1}\right),
$$

implying by (50) that

$$
Y_{\mathrm{ge}}\left(\mathcal{U}_{b}\right)=\int_{C_{n}} w(x) \mathbf{1}_{x, \mathrm{ge}}\left(\mathcal{U}_{b}\right) d x \leq Y_{\mathrm{ge}}\left(\mathcal{U}_{a}\right)+\pi_{p}|\mathbf{w}|(b-a)|\rho|^{p}
$$

for $b=a+1$, and hence for all $0 \leq a \leq b \leq m$. In particular,

$Y_{\mathrm{ge}}^{s}=Y_{\mathrm{ge}}\left(\mathcal{U}_{N+A}\right) \leq Y_{\mathrm{ge}}\left(\mathcal{U}_{N}\right)+\pi_{p}|\mathbf{w}| A|\rho|^{p} \leq Y_{\mathrm{ge}}\left(\mathcal{U}_{N}\right)+\pi_{p}|\mathbf{w}||\mathbf{d}||\rho|^{p}=Y_{\mathrm{ge}}+\pi_{p}|\mathbf{w}||\mathbf{d}||\rho|^{p}$,

thus verifying the claim for $Y_{\text {ge }}$. 
To handle $Y_{\text {ne }}$, with $N$ as before satisfying $\mathcal{L}(N)=\mathcal{L}\left(M\left(U_{0}, \mathcal{U}\right)\right)$, construct $A$ as in Part 3 of Lemma 2.1, so that $-1 \leq A \leq 1$ and

$$
\mathcal{L}(N+A)=\mathcal{L}\left(M\left(U_{0}\right) \mid M\left(U_{0}, \mathcal{U}\right) \neq d\left(U_{0}\right)\right)
$$

Arguing as before, we have that $Y_{\text {ne }}^{s}=Y_{\text {ne }}\left(\mathcal{U}_{N+A}\right)$ has the $Y_{\text {ne }}=Y\left(\mathcal{U}_{N}\right)$-size biased distribution. As the configurations $\mathcal{U}_{a}$ and $\mathcal{U}_{a+1}$ differ only at the point indexed by $\beta_{a}$, with $\Delta$ denoting the symmetric difference of sets, as in (59) one has

$$
\mathbf{1}_{x, \text { ne }}\left(\mathcal{U}_{a}\right) \leq \mathbf{1}_{x, \text { ne }}\left(\mathcal{U}_{b}\right)+\mathbf{1}\left(x \in B_{\beta_{a \wedge b}}^{a} \Delta B_{\beta_{a \wedge b}}^{b}\right) \quad \text { for }|a-b| \leq 1,\{a, b\} \subset[m] .
$$

As $|A| \leq 1$,

$$
Y_{\mathrm{ne}}^{s}=Y_{\mathrm{ne}}\left(\mathcal{U}_{N+A}\right) \leq Y_{\mathrm{ne}}\left(\mathcal{U}_{N}\right)+\pi_{p}|\mathbf{w}||A||\rho|^{p} \leq Y_{\mathrm{ne}}+\pi_{p}|\mathbf{w}||\rho|^{p},
$$

thus verifying the claim for $Y_{\text {ne }}$.

\subsubsection{Number of neighbors in germ-grain models}

In this section, we consider the occupancy model $\mathbf{M}(\mathcal{V})=\left(M_{\alpha}(\mathcal{V})\right)_{\alpha \in[m]}$, based on the configuration $\mathcal{V}=\left(v_{\alpha}\right)_{\alpha \in[m]}$, with components

$$
M_{\alpha}(\mathcal{V})=\sum_{\beta \in[m] \backslash\{\alpha\}} \mathbf{1}\left(B_{\alpha} \cap B_{\beta} \neq \varnothing\right),
$$

where $B_{\alpha}$ is the closed unit ball centered at $v_{\alpha}, \alpha \in[m]$. That is, $M_{\alpha}$ counts the number of neighbors of $v_{\alpha}$, where we say $v_{\alpha}$ and $v_{\beta}$ are neighbors, and write $v_{\alpha} \sim v_{\beta}$, when $\alpha \neq \beta$ and $B_{\alpha} \cap B_{\beta} \neq \varnothing$. The variables $Y_{\text {ge }}$ and $Y_{\text {ne }}$ are again given as in (42) for $\mathcal{V}=\mathcal{U}$, and are the weighted sums of the contributions from points $U_{\alpha}$ of $\mathcal{U}$ that have at least $d_{\alpha}$ neighbors, and a number other than $d_{\alpha}$ neighbors, respectively. We drop the dependence on $\mathcal{V}$ unless clarity demands it.

Suppressing the dimension $p$ in our notation, in this section, we specialize to the unit radius $\rho_{\alpha}=1$ case in order to allow Lemma 3.3 below to yield a bound on our coupling in simple terms of classical geometric constants related to the 'kissing numbers' $\kappa_{1}^{*}$; see [15] and [56]. With $B_{0}$ the closed unit ball of radius one centered at the origin, the constant $\kappa_{1}^{*}$ is the maximum number of closed unit balls in $\mathbb{R}^{p}$ that can be packed so that their closures intersect $B_{0}$, with all balls having disjoint interiors. The related constant $\kappa_{1}$ arises below, which is the maximum number of unit balls that can be packed so that they all intersect $B_{0}$, but are disjoint from each other. The value of $\kappa_{1}$ is a lower bound on $\kappa_{1}^{*}$. In two dimensions $\kappa_{1}=5$ and $\kappa_{1}^{*}=6$, though $\kappa_{1}=\kappa_{1}^{*}=12$ in three dimensions and it seems likely the equality holds much more generally. In the subsection, we assume $n$ is large enough so that

$$
\sqrt{p} n^{1 / p}>2 m \text { and } n^{1 / p}>6,
$$

the first inequality being (48) specialized to the unit radius case, and the second imposed so that Lemma 3.3 may be invoked over $C_{n}$. 
For $\mathcal{A} \subset\{1,2, \ldots\}$ and $\mathbf{d}=\left(d_{\alpha}\right)_{\alpha \in \mathcal{A}}$ a bounded collection of positive integers, let $\kappa_{\mathbf{d}}$ be the maximum value of $k \geq 0$ such that there exists $\Gamma \subset \mathcal{A}$ of size $k$ and a set of points $\mathcal{V}=\left(v_{\alpha}\right)_{\alpha \in \Gamma}$ in $\mathbb{R}^{p}$ such that

$$
B_{\alpha} \cap B_{0} \neq \varnothing \quad \text { and } \quad \sum_{\gamma \in \Gamma \backslash\{\alpha\}} 1\left(B_{\alpha} \cap B_{\gamma} \neq \varnothing\right)=d_{\alpha}-1 \quad \text { for all } \alpha \in \Gamma,
$$

with $v_{0}$ the origin, and $B_{\alpha}$ the unit ball centered at $v_{\alpha}$. That is, the constant $\kappa_{\mathbf{d}}$ is the maximum size of a set $\Gamma$ of points in $\mathbb{R}^{p}$ such that the number of neighbors of the points in the set $\Gamma$, among the points indexed by $\Gamma \cup\{0\}$, drops from $d_{\alpha}$ to $d_{\alpha}-1$ (increases from $d_{\alpha}-1$ to $d_{\alpha}$ ) upon the removal (insertion) of the unit ball at the origin. If $d_{\alpha}=1$ for all $\alpha \in \mathcal{A}$, then $\kappa_{\mathbf{d}}=\kappa_{1}$. Let $d_{(\alpha)}$ be the values of $d_{\alpha}$ in a non-strict decreasing order, that is,

$$
d_{(1)} \geq d_{(2)} \geq \cdots
$$

Lemma 3.3. In $\mathbb{R}^{p}$ for any dimension $p \geq 1$,

$$
\kappa_{\mathbf{d}} \leq \sigma_{\mathbf{d}} \quad \text { where } \sigma_{\mathbf{d}}=\sum_{\alpha \in\left[\kappa_{1}\right]} d_{(\alpha)},
$$

and the bound is achieved when there exists a pairwise disjoint collection of indices $\mathcal{Q}_{\alpha} \subset \mathcal{A}$, $\alpha \in\left[\kappa_{1}\right]$, with $\left|\mathcal{Q}_{\alpha}\right|=d_{(\alpha)}$ such that $d_{\beta}=d_{(\alpha)}$ for all $\beta \in \mathcal{Q}_{\alpha}$.

Proof. To show the upper bound, let $\mathcal{V}=\left(v_{\alpha}\right)_{\alpha \in \Gamma}, \Gamma \subset \mathcal{A}$, be an arbitrary collection of points in $\mathbb{R}^{p}$ such that, for all $\alpha \in \Gamma$, the closed unit ball around $v_{\alpha}$ intersects the closed unit ball around the origin and has exactly $d_{\alpha}-1$ neighbors in $\mathcal{V}$. As the numbers $\left(d_{\alpha}\right)_{\alpha \in \mathcal{A}}$ are bounded, the set $\mathcal{V}$ is finite. Let $\mathcal{R}$ be a subset of $\mathcal{V}$ of maximal size with the property that the closed unit balls centered at the points of $\mathcal{R}$ are pairwise disjoint. By the maximality of $\mathcal{R}$, each point in $\mathcal{V} \backslash \mathcal{R}$ must be a neighbor of at least one point in $\mathcal{R}$, implying that $\mathcal{V}$ is the union of $\mathcal{R}$ and those points of $\mathcal{V}$ that are a neighbor of some point of $\mathcal{R}$. Hence,

$$
\mathcal{V}=\bigcup_{v \in \mathcal{R}}\left(\{v\} \cup \bigcup_{w \in \mathcal{V}: w \sim v}\{w\}\right)
$$

As the point $v_{\alpha} \in \mathcal{V}$ has $d_{\alpha}-1$ neighbors in $\mathcal{V}$, and as $|\mathcal{R}|$ can be at most $\kappa_{1}$, we obtain

$$
|\mathcal{V}| \leq \sum_{\alpha=1}^{\kappa_{1}} d_{\alpha} \leq \sum_{\alpha=1}^{\kappa_{1}} d_{(\alpha)}=\sigma_{\mathbf{d}}
$$

Taking supremum over all such collections $\left(v_{\alpha}\right)_{\alpha \in \mathcal{A}}$ we obtain the inequality $\kappa_{\mathbf{d}} \leq \sigma_{\mathbf{d}}$.

We now show that the bound is achieved when $\mathbf{d}$ satisfies the given condition. By definition of $\kappa_{1}$, there exists a collection of points $v_{\alpha}, \alpha \in\left[\kappa_{1}\right]$, in $\mathbb{R}^{p}$ such that the closed unit balls $B_{\alpha}$, $\alpha \in\left[\kappa_{1}\right]$ around each point intersect the closed unit ball $B_{0}$ at the origin, but no other ball $B_{\beta}, \beta \in$ $\left[\kappa_{1}\right] \backslash\{\alpha\}$. Now consider the collection of $\sigma_{\mathbf{d}}$ unit balls consisting of $d_{(\alpha)}$ copies of the unit ball with center $u_{\alpha}$, for each $\alpha \in\left[\kappa_{1}\right]$. Each of the $d_{(\alpha)}$ balls with center at $u_{\alpha}$ has $d_{(\alpha)}$ neighbors 
when the closed unit ball at the origin is included, but $d_{(\alpha)}-1$ neighbors when it is not. Hence for such $\mathbf{d}$, we achieve $\kappa_{\mathbf{d}} \geq \sum_{\alpha=1}^{\kappa_{1}} d_{(\alpha)}=\sigma_{\mathbf{d}}$.

We prove concentration for the neighborhood counts $Y_{\text {ge }}$ and $Y_{\text {ne }}$ by showing that bounded size bias couplings for these variables exist with respective bounds

$$
c_{\mathrm{ge}}=|\mathbf{w}||\mathbf{d}|\left(\sigma_{\mathbf{d}}+1\right) \quad \text { and } \quad c_{\mathrm{ne}}=|\mathbf{w}|\left(\sigma_{\mathbf{d}}+\sigma_{\mathbf{d}+1}+1\right),
$$

where $|\mathbf{d}|$ and $|\mathbf{w}|$ are as in (25) for given threshold and weight vectors, and where $\mathbf{d}+1$ denotes the vector $\left(d_{\alpha}+1\right)_{\alpha \in[m]}$.

Noting that the case $m=1$ is trivial, for $m=2$ we have $Y_{\mathrm{ge}} \leq 2|\mathbf{w}|$, hence $Y_{\mathrm{ge}}^{s}$ is also so upper bounded, and the inequality $Y_{\mathrm{ge}}^{s} \leq Y_{\mathrm{ge}}+c_{\mathrm{ge}}$ holds trivially, with similar remarks applying to $Y_{\mathrm{ne}}$. Hence, we may assume in the remainder of this section that $m \geq 3$. Under (62) it is not difficult to see that the constant $\kappa_{1}$ computed over $C_{n}$ is the same as that over $\mathbb{R}^{p}$, and Lemma 3.3 holds over $C_{n}$ as well.

For $\beta \neq \alpha$ and $x \in C_{n}$, letting

$$
p_{\beta}(x)=P\left(D\left(x, U_{\beta}\right) \leq 2\right),
$$

we have that the conditional law $\mathcal{L}\left(M_{\alpha} \mid U_{\alpha}=x\right)$ is Poisson $\operatorname{Binomial} \mathcal{P B}\left(\mathbf{p}_{\alpha}(u)\right)$ where $\mathbf{p}_{\alpha}(u)=$ $\left(p_{\beta}(u)\right)_{\beta \in[m] \backslash\{\alpha\}}$, and $Y_{\text {ge }}$ and $Y_{\text {ne }}$ have expectations given respectively by

$$
\begin{aligned}
& \mu_{\mathrm{ge}}=\sum_{\alpha \in[m]} w_{\alpha} \int_{C_{n}} q_{\mathrm{ge}}\left(d_{\alpha}, \mathbf{p}_{\alpha}(u)\right) f_{\alpha}(u) d u \quad \text { and } \\
& \mu_{\mathrm{ne}}=\sum_{\alpha \in[m]} w_{\alpha} \int_{C_{n}} q_{\mathrm{ne}}\left(d_{\alpha}, \mathbf{p}_{\alpha}(u)\right) f_{\alpha}(u) d u .
\end{aligned}
$$

Theorem 3.3. Concentration of measure inequalities (5)-(8) hold for all $m \geq 1$ for $Y_{\mathrm{ge}}$ and $Y_{\mathrm{ne}}$ as in (42), computed on the neighbor count vector (61) for the germ-grain model, with corresponding $\mu$ and $c$ given respectively, by (65) and (63).

Proof. First, we show that there exists a coupling of $Y_{\mathrm{ge}}$ to $Y_{\mathrm{ge}}^{s}$, having the $Y_{\mathrm{ge}}$-size biased distribution, that satisfies $Y_{\mathrm{ge}}^{s} \leq Y_{\mathrm{ge}}+c_{\mathrm{ge}}$. To do so, for each $\alpha \in[m]$ we apply the reasoning in the proof of Lemma 3.2, replacing $m$ and $U_{0}$ there by $m-1$ and $U_{\alpha}$. In particular, upon that replacement (57) becomes

$$
\begin{aligned}
& \mathcal{L}\left(\mathcal{U} \mid M_{\alpha}(\mathcal{U}) \geq d_{\alpha}\right)=\int_{C_{n}} \mathcal{L}\left(\mathcal{U} \mid M_{\alpha, u}(\mathcal{U}) \geq d_{\alpha}\right) \tilde{f}_{\alpha}(u) d u \\
& \text { where } \tilde{f}_{\alpha}(u)=\frac{P\left(M_{\alpha, u}(\mathcal{U}) \geq d_{\alpha}\right)}{P\left(M_{\alpha}(\mathcal{U}) \geq d_{\alpha}\right)} f_{\alpha}(u)
\end{aligned}
$$

where $M_{\alpha, u}(\mathcal{U})$ is given by (61) with $B_{\alpha}$ replaced by the unit ball centered at $u \in C_{n}$. Hence, we sample $\widetilde{U}_{\alpha}$ on $C_{n}$ with density $\widetilde{f}_{\alpha}(u)$, and for $\widetilde{U}_{\alpha}=u$, construct a configuration $\mathcal{U}$ with law $\mathcal{L}\left(\mathcal{U} \mid P\left(M_{\alpha, u}(\mathcal{U}) \geq d_{\alpha}\right)\right)$ in order to achieve $\mathcal{L}\left(\mathcal{U} \mid P\left(M_{\alpha}(\mathcal{U}) \geq d_{\alpha}\right)\right)$. 
Continuing to follow the proof of Lemma 3.2, for each $\alpha \in[m]$ and $a \in[m-1]_{0}$ we obtain coupled configurations

$$
\mathcal{U}_{a}^{\alpha}=\left\{U_{\beta, a}^{\alpha}, \beta \in[m]\right\} \quad \text { satisfying } \quad \mathcal{L}\left(\mathcal{U}_{a}^{\alpha}\right)=\mathcal{L}\left(\mathcal{U} \mid M_{\alpha}=a\right),
$$

where $U_{\alpha, a}^{\alpha}=u$ for all $\alpha \in[m]$, and where $\mathcal{U}_{a}^{\alpha}$ and $\mathcal{U}_{a+1}^{\alpha}$ differ in only one point indexed by, say, $\beta_{\alpha}$, where $\beta_{\alpha} \neq \alpha$. We will say $U_{\beta_{a}, a}^{\alpha}$ was removed from the configuration, into which $U_{\beta_{a}, a+1}^{\alpha}$ is inserted.

Let $N_{\alpha, u}$ be constructed on this same space with distribution $\mathcal{L}\left(M_{\alpha, u}(\mathcal{U})\right)$. Applying Corollary 2.1 to $M_{u, \alpha}=M_{\alpha, u}(\mathcal{U})$, we obtain $A_{\alpha, u}$ satisfying

$$
\mathcal{L}\left(N_{\alpha, u}+A_{\alpha, u}\right)=\mathcal{L}\left(M_{\alpha, u} \mid M_{\alpha, u} \geq d_{\alpha}\right) \quad \text { with } 0 \leq A_{\alpha, u} \leq d_{\alpha} .
$$

Recalling that $u$ was chosen with density $\widetilde{f}_{\alpha}(u)$, and letting $N_{\alpha}$ and $A_{\alpha}$ denote $N_{\alpha, u}$ and $A_{\alpha, u}$ respectively for notational simplicity, by $\mathcal{L}\left(N_{\alpha}\right)=\mathcal{L}\left(M_{\alpha, u}(\mathcal{U})\right)$ and (66), as in Lemma 3.2 we have

$$
\mathcal{L}\left(\mathcal{U}_{N_{\alpha}}^{\alpha}\right)=\mathcal{L}(\mathcal{U}) \quad \text { and } \quad \mathcal{L}\left(\mathcal{U}_{N_{\alpha}+A_{\alpha}}^{\alpha}\right)=\mathcal{L}\left(\mathcal{U} \mid M_{\alpha}(\mathcal{U}) \geq d_{\alpha}\right)
$$

where $\mathcal{U}_{N_{\alpha}}^{\alpha}$ and $\mathcal{U}_{N_{\alpha}+A_{\alpha}}^{\alpha}$ are configurations corresponding to $N_{\alpha}$ and $N_{\alpha}+A_{\alpha}$, respectively.

Let $\mathbf{N}_{a}^{\alpha}=\left(N_{\beta, a}^{\alpha}\right)_{\beta \in[m]}$ be the occupancy counts corresponding to $\mathcal{U}_{a}^{\alpha}$ and write $Y_{\text {ge }}(\mathcal{V})$ for (42) evaluated on the configuration $\mathcal{V}$. By the second identity in (68), the indicators $X_{\beta}^{\alpha}=$ $\mathbf{1}\left(N_{\beta, N_{\alpha}+A_{\alpha}}^{\alpha} \geq d_{\beta}\right)$ satisfy (10) with $X_{\beta}=\mathbf{1}\left(N_{\beta, N_{\alpha}}^{\alpha} \geq d_{\beta}\right)$. Hence, by Lemma 1.1 , with $I$ independent of all other variables with distribution (12), $Y_{\text {ge }}^{s}=Y_{\text {ge }}\left(\mathcal{U}_{N_{I}+A_{I}}^{I}\right)$ has the $Y_{\text {ge }}=Y\left(\mathcal{U}_{N_{I}}^{I}\right)$ size biased distribution. The first identity in (68) shows that $\mathcal{L}\left(\mathcal{U}_{N_{I}}^{I}\right)=\mathcal{L}(\mathcal{U})$.

As the removal of $U_{\beta_{a}, a}^{\alpha}$ from $\mathcal{U}_{a}^{\alpha}$ can only decrease the number of its neighbors in the configuration $\mathcal{U}_{a+1}^{\alpha}$, increases in occupancy counts for the configuration $\mathcal{U}_{\beta_{a}, a+1}^{\alpha}$ over their values in $\mathcal{U}_{a}^{\alpha}$ can occur only for the point $U_{\beta_{a}, a+1}^{\alpha}$, taking the place of $U_{\beta_{a}, a}^{\alpha}$, and its set of neighbors. However, with $U_{\beta_{a}, a+1}^{\alpha}$ playing the role of the origin in Lemma 3.3, its insertion into the point set $\left\{U_{\beta, a}^{\alpha}: \beta \neq \beta_{a}\right\}$ can increase at most $\sigma_{\mathbf{d}}$ of the counts $N_{\beta, a}^{\alpha}, \beta \neq \beta_{a}$, of former value $d_{\beta}-1$ to counts $N_{\beta, a+1}^{\alpha}$ of value $d_{\beta}$. Hence, also accounting for the possible change of the count at the point indexed by $\beta_{a}$, we have

$$
\left|\mathcal{N}_{a+1}\right| \leq \sigma_{\mathbf{d}}+1 \quad \text { where } \mathcal{N}_{a+1}=\left\{\beta \in[m]: \mathbf{1}\left(N_{\beta, a}^{\alpha} \geq d_{\beta}\right)=0, \mathbf{1}\left(N_{\beta, a+1}^{\alpha} \geq d_{\beta}\right)=1\right\},
$$

and therefore

$$
\begin{aligned}
\sum_{\beta \in[m]} w_{\beta} \mathbf{1}\left(N_{\beta, a+1}^{\alpha} \geq d_{\beta}\right) & =\sum_{\beta \notin \mathcal{N}_{a+1}} w_{\beta} \mathbf{1}\left(N_{\beta, a+1}^{\alpha} \geq d_{\beta}\right)+\sum_{\beta \in \mathcal{N}_{a+1}} w_{\beta} \mathbf{1}\left(N_{\beta, a+1}^{\alpha} \geq d_{\beta}\right) \\
& \leq \sum_{\beta \notin \mathcal{N}_{a+1}} w_{\beta} \mathbf{1}\left(N_{\beta, a}^{\alpha} \geq d_{\beta}\right)+|\mathbf{w}|\left|\mathcal{N}_{a+1}\right| \\
& \leq \sum_{\beta \in[m]} w_{\beta} \mathbf{1}\left(N_{\beta, a}^{\alpha} \geq d_{\beta}\right)+|\mathbf{w}|\left(\sigma_{\mathbf{d}}+1\right)
\end{aligned}
$$


Now, by (67),

$$
Y_{\mathrm{ge}}\left(\mathcal{U}_{N_{\alpha}+A_{\alpha}}^{\alpha}\right) \leq Y\left(\mathcal{U}_{N_{\alpha}}^{\alpha}\right)+|\mathbf{w}| A_{\alpha}\left(\sigma_{\mathbf{d}}+1\right) \leq Y\left(\mathcal{U}_{N_{\alpha}}^{\alpha}\right)+|\mathbf{w}||\mathbf{d}|\left(\sigma_{\mathbf{d}}+1\right),
$$

and mixing over $\alpha$ yields

$$
Y_{\mathrm{ge}}^{S}=Y_{\mathrm{ge}}\left(\mathcal{U}_{N_{I}+A_{I}}^{I}\right) \leq Y\left(\mathcal{U}_{N_{I}}^{I}\right)+|\mathbf{w}||\mathbf{d}|\left(\sigma_{\mathbf{d}}+1\right)=Y_{\mathrm{ge}}+|\mathbf{w}||\mathbf{d}|\left(\sigma_{\mathbf{d}}+1\right),
$$

verifying the claim for $Y_{\mathrm{ge}}$.

Next, we show that there exists a coupling of $Y_{\text {ne }}$ to $Y_{\text {ne }}^{s}$, having the $Y_{\text {ne }}$-size biased distribution, satisfying $Y_{\mathrm{ne}}^{s} \leq Y_{\mathrm{ne}}+c_{\mathrm{ne}}$, where $c_{\mathrm{ge}}$ and $c_{\mathrm{ne}}$ are given by (63). The construction for $Y_{\mathrm{ne}}$ will be similar, the only difference being that the initial removal of $U_{\beta_{a}, a}^{\alpha}$ can cause $\sigma_{\mathbf{d}}$ counts to drop from $d_{\alpha}$ to $d_{\alpha}-1$, while the insertion of $U_{\beta_{a}, a+1}^{\alpha}$ can cause $\sigma_{\mathbf{d}+1}$ counts of value $d_{\alpha}$ to rise to $d_{\alpha}+1$. Hence in this case we obtain, as claimed,

$$
\sum_{\beta \in[m]} w_{\beta} \mathbf{1}\left(N_{\beta, a+1}^{\alpha} \neq d_{\beta}\right) \leq \sum_{\beta \in[m]} w_{\beta} \mathbf{1}\left(N_{\beta, a}^{\alpha} \neq d_{\beta}\right)+|\mathbf{w}|\left(\sigma_{\mathbf{d}}+\sigma_{\mathbf{d}+1}+1\right) .
$$

When all points are uniformly distributed over $C_{n}, f_{\beta}(u)=1 / n$ and the probability $p_{\beta}(u)$ in (64) is the constant $2^{p} \pi_{p} / n$ for all $u \in C_{n}$, where $\pi_{p}$ is the volume of the unit ball in dimension $p$. Hence with weights $w_{\alpha}=1$ for all $\alpha \in[m]$, we obtain

$$
\mu_{\mathrm{ge}}=m P\left(M_{\alpha} \geq d\right)=m P\left(\operatorname{Bin}\left(m-1,2^{p} \pi_{p} / n\right) \geq d\right)
$$

with similar remarks applying to $\mu_{\text {ne }}$.

\subsection{Multinomial occupancy}

Among the many applications of multinomial occupancy models, in which $n$ balls are distributed independently to $m$ urns (see [35] for an overview), are the well-known species trapping problem (see [11,45], or [50]) and the closely-related problem of statistical linguistics (see [20] and [54]). The study of the number of empty urns, or equivalently the $d=1$ case of $Y_{\text {ge }}$ in (2), was initiated in [44] and [55] where it was shown that the properly standardized distribution of $Y_{\mathrm{ge}}$ is asymptotically normal when balls land in urns uniformly. Bounds in the $L^{\infty}$ metric between the standard normal distribution and standardized finite sample distribution of the $d=1$ case of $Y_{\mathrm{ge}}$ was provided by [22] in the uniform case, for $Y_{\text {eq }}$ by [43] in the uniform and some non-uniform cases, and for all $d \geq 2$ for $Y_{\text {eq }}$ by [8] in the uniform case. Concentration of measure inequalities for the number of empty urns were obtained in [18] by exploiting negative association, discussed in Section 4.2.

For $\alpha \in[m]$ let the component $M_{\alpha}$ of the vector $\mathbf{M}=\left(M_{\alpha}\right)_{\alpha \in[m]}$ count the number of balls in urn $\alpha$ when $n$ balls are independently distributed into $m$ urns and for $j \in[n]$ the location $L_{j}$ of ball $j$ is urn $\alpha$ with probability $p_{\alpha, j}$. In particular,

$$
M_{\alpha}=\sum_{j=1}^{n} \mathbf{1}\left(L_{j}=\alpha\right)
$$


As in Section 3.1, we may assume that $p_{\alpha, j}<1$ for all $(\alpha, j) \in[m] \times[n]$, that $\sum_{j=1}^{n} p_{\alpha, j}>0$ for all urns $\alpha \in[m]$, and that each of the summand indicators of $Y_{\mathrm{ge}}$ and $Y_{\mathrm{ne}}$ in (42) is nontrivial. With $\mathbf{p}_{\alpha}=\left(p_{\alpha, j}\right)_{j \in[n]}$ we have $M_{\alpha} \sim \mathcal{P B}\left(\mathbf{p}_{\alpha}\right)$ and, arguing as before, the means $\mu_{\mathrm{ge}}$ and $\mu_{\mathrm{ne}}$ again have the form (43).

We may now summarize the main result of this subsection.

Theorem 3.4. Concentration of measure inequalities (5)-(8) hold for all $m \geq 1$ for multinomial occupancy counts,

1. with $Y_{\mathrm{ge}}, \mu_{\mathrm{ge}}$ and $c_{\mathrm{ge}}$ given by (42), (43), and $|\mathbf{w}|$,

2. with $Y_{\mathrm{ne}}, \mu_{\mathrm{ne}}$ and $c_{\mathrm{ne}}$ given by (42), (43) and $2|\mathbf{w}|$.

Theorem 3.4 will follow immediately from Theorem 1.1 from the coupling construction provided by the next lemma.

Lemma 3.4. In the multinomial occupancy model there exists a coupling of $Y_{\mathrm{ge}}$ to $Y_{\mathrm{ge}}^{s}$, having the $Y_{\mathrm{ge}}$-size biased distribution, that satisfies $Y_{\mathrm{ge}}^{s} \leq Y_{\mathrm{ge}}+|\mathbf{w}|$, and a coupling of $Y_{\mathrm{ne}}$ to $Y_{\mathrm{ne}}^{s}$, having the $Y_{\mathrm{ne}}$-size biased distribution, satisfying $Y_{\mathrm{ne}}^{s} \leq Y_{\mathrm{ne}}+2|\mathbf{w}|$.

Proof. The reasoning of Lemma 3.1 applies with only minimal changes. We take configurations in this model to be

$$
\mathcal{U}=\left\{L_{j}, j \in[n]\right\},
$$

the collection of locations of all $n$ balls. As for each $\alpha \in[m]$ the corresponding count $M_{\alpha}$ has a LC marginal distribution with support $\mathcal{S}_{\alpha}$ satisfying inf $\mathcal{S}_{\alpha}=0$, in order to invoke Theorem 2.1 it is only required to show that, for all $\alpha \in[m]$, configurations $\left\{\mathcal{U}_{a}^{\alpha}, a \in[m]\right\}$ exist with Properties $(0, \geq)$ and $(1, \neq)$ satisfying $(32)$.

As in Lemma 3.1, through the use of Lemma 2.4, for each $\alpha \in[m]$ we obtain configurations $\mathcal{U}_{a}^{\alpha}$ with corresponding counts $\mathbf{N}_{a}^{\alpha}=\left(N_{\beta, a}^{\alpha}\right)_{\beta \in[m]}$ such that

$$
\mathcal{L}\left(\mathcal{U}_{a}^{\alpha}\right)=\mathcal{L}\left(\mathcal{U} \mid M_{\alpha}=a\right) \quad \text { for all } a \in[n]_{0}
$$

and, for all $a \in[n-1]_{0}, \mathcal{U}_{a+1}^{\alpha}$ differs from $\mathcal{U}_{a}^{\alpha}$ by a single element, indexed by $j_{a}$, say. As $N_{\alpha, a}^{\alpha}=a$ for all $a \in[n]_{0}$, we must have that $L_{j_{a}, a}^{\alpha} \neq \alpha$ and $L_{j_{a}, a+1}^{\alpha}=\alpha$, where $L_{j, a}^{\alpha}$ denotes the location of ball $j$ in configuration $\mathcal{U}_{a}^{\alpha}$. In particular, $N_{\beta, a+1}^{\alpha} \leq N_{\beta, a}^{\alpha}$ for all $\beta \neq \alpha$, and so our observation in (29) guarantees that the occupancy counts corresponding to $\left\{\mathcal{U}_{a}^{\alpha}, a \in[m]\right\}$ have Property $(0, \geq)$.

For $Y_{\text {ne }}$, with $\{a, b\} \subset \mathcal{S}_{\alpha}$ satisfying $|b-a|=1$, we note that the counts corresponding to the configurations $\mathcal{U}_{a}^{\alpha}$ and $\mathcal{U}_{b}^{\alpha}$ differ only at two indices, one of which is $\alpha$. Hence, these counts have Property $(1, \neq)$ by $(28)$.

In the asymptotic regime most studied, balls are uniformly distributed, thresholds are constant and the weights are taken to be identically 1 . That is, $p_{\alpha, j}=1 / m, w_{\alpha}=1$ and $d_{\alpha}=d$ for each $\alpha \in[m]$ and $j \in[n]$. For this special case, the expectations in (43) simplify to

$$
\mu_{\mathrm{ge}}=m P(\operatorname{Bin}(n, 1 / m) \geq d) \quad \text { and } \quad \mu_{\mathrm{ne}}=m(1-P(\operatorname{Bin}(n, 1 / m)=d)),
$$


and the concentration bounds obtained via size biasing can be used for $Y_{\mathrm{ge}}$ with $c=1$, and for $Y_{\text {ne }}$ with $c=2$.

The expectations in (70) are of a form similar to those of (45) for the standard Erdôs-Rényi model. Thus, by arguing as in Section 3.1 we can study in the same manner the behavior of the bounds obtained here.

Remark 3.1 (Multivariate hypergeometric sampling). The techniques used here to construct a bounded coupling can be modified to obtain concentration inequalities for another relevant model where we have negative association. Let $n$ be the sum of the given positive integers $\left(n_{\alpha}\right)_{\alpha \in[m]}$, and consider an urn containing $n$ colored balls, $n_{\alpha}$ of which are of color $\alpha$. For $s \in[n]_{0}$ let $M_{\alpha}$ be the number of balls of color $\alpha$ obtained upon sampling $s$ distinct balls uniformly from the urn without replacement, and set $\mathbf{M}=\left(M_{\alpha}\right)_{\alpha \in[m]}$. Let $Y_{\text {ge }}$ and $Y_{\text {ne }}$ be as in (42).

Then, for all $\alpha \in[m]$ the distribution of $M_{\alpha}$ is hypergeometric, and the expected values of $Y_{\mathrm{ge}}$ and $Y_{\text {ne }}$ are, respectively,

$$
\mu_{\mathrm{ge}}=\sum_{\alpha \in[m]} w_{\alpha} \sum_{j \geq d_{\alpha}} q\left(j ; n_{\alpha}, s, n\right) \quad \text { and } \quad \mu_{\mathrm{ne}}=\sum_{\alpha \in[m]} w_{\alpha} \sum_{j \neq d_{\alpha}} q\left(j ; n_{\alpha}, s, n\right),
$$

where

$$
q(j ; k, \ell, i)=\left(\begin{array}{l}
k \\
j
\end{array}\right)\left(\begin{array}{l}
i-k \\
\ell-j
\end{array}\right) /\left(\begin{array}{l}
i \\
\ell
\end{array}\right)
$$

for values of $j, k, \ell, i$ such that the quotient (72) is defined, setting $q(j ; k, \ell, i)=0$ otherwise. Moreover, it is shown in [21], Theorem A, that a hypergeometric random variable can be written as a sum of independent but non-identically-distributed Bernoulli random variables, from which we may conclude that the hypergeometric distribution is LC. Our techniques above then can be modified to show that there exists a coupling of $Y_{\mathrm{ge}}$ to $Y_{\mathrm{ge}}^{s}$, having the $Y_{\mathrm{ge}}$-size biased distribution, that satisfies $Y_{\mathrm{ge}}^{s} \leq Y_{\mathrm{ge}}+|\mathbf{w}|$, and a coupling of $Y_{\mathrm{ne}}$ to $Y_{\mathrm{ne}}^{s}$, having the $Y_{\mathrm{ne}}$-size biased distribution, satisfying $Y_{\text {ne }}^{s} \leq Y_{\text {ne }}+2|\mathbf{w}|$. These couplings provide concentration of measure inequalities where the means are given by (71). We omit the details.

\section{Comparisons}

In this section, we compare our results to concentration bounds obtained by other means. Our comparisons will be with the following three well-known techniques: (i) McDiarmid's Inequality, (ii) Use of negative association and (iii) Self Bounding and Certifiable functions. Of these three, the last technique is the most comparable. For simplicity and concreteness, in most of our comparisons below we will consider

$$
Y_{\mathrm{ge}}=\sum_{\alpha \in[m]} \mathbf{1}\left(M_{\alpha} \geq d\right)
$$

with unit weighting and constant threshold count. 


\subsection{McDiarmid's inequality}

One of the most useful concentration results is the McDiarmid, or bounded difference, inequality which is a consequence of the Azuma-Hoeffding bound; see [5,30] and [40]. The inequality applies to quantities $Y$ that can be expressed as a function $f\left(X_{1}, \ldots, X_{n}\right)$ of independent random variables $X_{1}, \ldots, X_{n}$ when, for all $i \in[n]$, there exists a constant $c_{i}$ such that

$$
\sup _{x_{i}, x_{i}^{\prime}}\left|f\left(x_{1}, \ldots, x_{i}, \ldots, x_{n}\right)-f\left(x_{1}, \ldots, x_{i}^{\prime}, \ldots, x_{n}\right)\right| \leq c_{i} .
$$

Under these conditions, the inequality provides the right tail bound

$$
P(Y-E Y \geq t) \leq \exp \left(-\frac{2 t^{2}}{\sum_{i=1}^{n} c_{i}^{2}}\right),
$$

and a corresponding left tail bound.

Although the bounded difference inequality is powerful and easy to apply, the quantity $\sum_{i=1}^{n} c_{i}^{2}$ on which it depends, obtained by taking supremums in (74) to estimate the worst-case behavior of $f$, may not accurately reflect the concentration properties of $f$.

To take the simplest example, let $Y$ have the Binomial distribution $\operatorname{Bin}(n, p)$. As $Y$ can be written as the sum of independent Bernoullis, inequality (74) is satisfied with $c_{i}=1$ and (75) yields

$$
P(Y-n p \geq t) \leq \exp \left(-\frac{2 t^{2}}{n}\right)
$$

However, for the Binomial it is known (see [41], for instance) that the true decay rate is $\exp \left(-t^{2} / 2 n p\right)$. In particular, use of (76) may not be adequate in situations where $p$ is small.

Applying Lemma 1.1 to $Y$, represented as an independent sum of indicators, we find that $Y^{s}$ can be formed by replacing any of the summand indicators by 1 , yielding $Y^{s} \leq Y+1$. Hence, the bound (8) yields

$$
P(Y-n p \geq t) \leq \exp \left(-\frac{t^{2}}{2(n p+t / 3)}\right) \quad \text { for all } t>0,
$$

which, specializing to the case $p \in(0,1 / 4)$, improves on the Azuma-Hoeffding bound (76) in the range $0<t<3 n(1 / 4-p)$, with upper range increasing to $(0, \infty)$ as $n \rightarrow \infty$.

We now turn to the standard Erdôs-Rényi random graph $\mathcal{G}_{m}$ on $m$ vertices with fixed edge probabilities $p$, as considered in Section 3.1, and let $Y_{\mathrm{ge}}$ be given by (73) where $M_{\alpha}$ is the degree of vertex $\alpha$. Clearly $Y_{\text {ge }}$ can be written as a function $f$ of $n=\left(\begin{array}{c}m \\ 2\end{array}\right)$ independent indicators $X_{1}, \ldots, X_{n}$, where $X_{i}$ denotes the presence of a given edge with respect to some fixed labeling. As a change in any $X_{i}$ affects the degree of exactly two vertices, $f$ satisfies the bounded differences condition (74) with $c_{i}=2$ for each $i=1, \ldots, n$. Hence, (75) and the complementary left tail inequality yield

$$
\max \left\{P\left(Y_{\mathrm{ge}}-\mu_{\mathrm{ge}} \leq-t\right), P\left(Y_{\mathrm{ge}}-\mu_{\mathrm{ge}} \geq t\right)\right\} \leq \exp \left(-\frac{t^{2}}{m(m-1)}\right),
$$


where $\mu_{\mathrm{ge}}$ is given by (45). Comparing the left tail bounds of (77) with (46), we see the size bias bound is preferred when

$$
\mu_{\mathrm{ge}} \leq m(m-1) /(2 d+2) .
$$

As $\mu_{\mathrm{ge}} \leq m$ due to $Y$ being the sum of $m$ indicators, this inequality is always satisfied for $m \geq 2 d+3$, and we see that the order of the exponent is improved from $O\left(-t^{2} / m^{2}\right)$ to $O\left(-t^{2} / m\right)$. Similar improvements will also hold for the Erdôs-Rényi type graph models with inhomogeneous edge probabilities, which, depending on their values, can become even more significant.

\subsection{Negative association}

Negative association has been used successfully to obtain concentration of measure inequalities for occupancy models. We recall from [32] (see also [18] and [48]) that a family of random variables $X_{1}, X_{2}, \ldots, X_{m}$ is said to be negatively associated if for any disjoint subsets $A_{1}, A_{2} \subset$ $[m]$,

$$
E\left(f\left(X_{i} ; i \in A_{1}\right) g\left(X_{j} ; j \in A_{2}\right)\right) \leq E\left(f\left(X_{i} ; i \in A_{1}\right)\right) E\left(g\left(X_{j} ; j \in A_{2}\right)\right)
$$

whenever $f$ and $g$ are coordinate-wise nondecreasing functions for which these expectations exist.

For the multinomial occupancy model of Section 3.3, the results of [32] show that the indicator summands $\mathbf{1}\left(M_{\alpha} \geq d_{\alpha}\right)$ of $Y_{\text {ge }}$ are negatively associated. Referring to Proposition 7 of [18], when $X_{1}, X_{2}, \ldots, X_{m}$ are negatively associated indicators, the random variable $Y=\sum_{i=1}^{m} X_{i}$ satisfies the right tail bound of (7), and hence bounds (5) and (8) with $c=1$. Thus, for this case, the estimates obtained for the right tail via negative association are at least as good as the ones that are obtained by using size biasing, with the same holding for the left tail. Indeed, Chernoff's bound for sums of independent random variables ([9], page 24) remains true for negatively associated sums, indicating that one may have strict improvements over the size bias method.

However, none of the other statistics discussed here can be handled using negative association. For instance, one cannot use negative association for our applications to random graphs and germ-grain models in Sections 3.1 and 3.2. In particular, for the standard Erdős-Rényi random graph, a simple application of Harris' inequality shows that the summand variables of $Y_{\mathrm{ge}}$ are positively associated.

Moreover, even for the multinomial occupancy model where negative association can be used for $Y_{\mathrm{ge}}$, the indicator summands in the multinomial occupancy count

$$
Y_{\mathrm{ne}}=\sum_{\alpha \in[m]} \mathbf{1}\left(M_{\alpha} \neq d_{\alpha}\right)
$$

are not negatively associated when the thresholds $d_{\alpha}$ are not all 0 . Hence, the method of [18] no longer applies, while the methods in this paper are still valid. For instance, when $d_{\alpha}=1$ for each $\alpha \in[m]$ and balls are distributed uniformly, Part 2 of Theorem 3.4 yields that (7) holds with 
$c=2$ and

$$
\mu_{\mathrm{ne}}=m\left(1-P\left(M_{1}=1\right)\right)=m\left(1-\frac{n}{m}\left(1-\frac{1}{m}\right)^{n-1}\right) .
$$

\subsection{Self-bounding and certifiable functions}

We have seen above that bounds produced by size biasing may improve on the bound (75) obtained using the bounded difference inequality as it replaces the $\operatorname{sum} \sum_{i=1}^{n} c_{i}^{2}$ by some function of the mean of $Y$. Bounds produced by the method of self bounding functions [41], of which certifiable functions are a special case, also have this advantage. We focus on the latter, as it is more straightforward to address the applications studied here in the framework of certifiable functions.

We begin by recalling the relevant definitions and results on certifiable functions from [41]; see also [9]. Let $c>0, a \geq 0$, and $b$ be given, and let a nonnegative measurable function $f$ on the product space $\Omega=\Pi_{i=1}^{n} \Omega_{i}$ satisfy the following two conditions.

(i) For each $x \in \Omega$, changing any coordinate $x_{j}$ changes the value of $f(x)$ by at most $c$.

(ii) If $f(x)=s$ then there is a set of coordinates $C \subset[n]$ of size at most $a s+b$ that certifies $f(x) \geq s$. That is, if the coordinates $i \in C$ of $y \in \Omega$ agree with those of $x$, then $f(y) \geq s$.

Let $X_{1}, \ldots, X_{n}$ be independent random variables with $X_{i}$ taking values in $\Omega_{i}, Y=$ $f\left(X_{1}, \ldots, X_{n}\right)$ where $f$ satisfies (i) and (ii) above, and $\mu=E Y$. Then for all $t \geq 0$,

$$
\begin{gathered}
P(Y-\mu \leq-t) \leq \exp \left(-\frac{t^{2}}{2 c^{2}(a \mu+b+t / 3 c)}\right) \quad \text { and } \\
P(Y-\mu \geq t) \leq \exp \left(-\frac{t^{2}}{2 c^{2}(a \mu+b+a t)}\right) .
\end{gathered}
$$

Before moving to a discussion of specific examples, we note that the asymptotic Poisson order $O(\exp (-t \log t))$ as $t \rightarrow \infty$ of the bound (7) with $c=1$ and $\mu=1$, is superior to the order $O(\exp (-t))$ of the bound (78) with $c=1$ and $a=1 / 2$, say, with similar types of improvement in order holding for other choices of constants. The order of the bounds achieved by certifiable functions, and self bounding functions more generally, seems to be intrinsic. Regarding using the entropy method to prove concentration inequalities for self bounding functions, via log Sobolev inequalities in particular, the authors of [9] note after the proof of Theorem 6.21 that, 'At least for $a>1$, there is no hope to derive Poissonian bounds... for the upper tail'.

To focus on a specific example, consider the random graph model of Section 3.1, and let $Y_{\text {ge }}$ be given by (73) where $M_{\alpha}$ is the degree of vertex $\alpha$ and $d \geq 2$. One can now easily show that the statistic $Y_{\text {ge }}$ is certifiable with $c=2, a=d$ and $b=0$, though both the lower and upper tail bounds (5) and (8) are superior to those obtained via (78).

Finally, we note that the Poisson tail concentration of measure inequalities of Theorem 1.1 will always provide further improvements over the bounds (5) and (8) applied in the previous paragraphs. However, the form of these latter bounds, being simpler than that of (7), allow for an easier comparison with (78), and although they are not the strongest bounds of those produced by the size bias method, they still suffice to demonstrate the improvements claimed. 


\section{Appendix: Monotonicity assumption}

We prove that the monotonicity assumption that $Y^{s} \geq Y$ assumed in [23] and [24] for the left tail bound can be removed, and that only $Y^{s} \leq Y+c$ is required for (5). First, we may assume that $Y$ is not almost surely constant as inequality (5) is trivially satisfied in that case. Since $Y \geq 0$ a.s., for all $\theta<0$ the moment generating function $m(\theta)=E\left(e^{\theta Y}\right)$ of $Y$ exists in an open interval containing $\theta$ and is differentiable at $\theta$. Differentiating under the expectation by dominated convergence and then applying the characterization of the size bias distribution (4), followed by an application of the inequality $1+x \leq e^{x}$, we obtain

$$
\begin{aligned}
m^{\prime}(\theta) & =E\left(Y e^{\theta Y}\right)=\mu E\left(e^{\theta Y^{s}}\right)=\mu E\left(e^{\theta Y} e^{\theta\left(Y^{s}-Y\right)}\right) \\
& \geq \mu E\left(e^{\theta Y}\left(1+\theta\left(Y^{s}-Y\right)\right)\right) \geq \mu E\left(e^{\theta Y}(1+\theta c)\right)=\mu(1+\theta c) m(\theta),
\end{aligned}
$$

where we have used $Y^{s}-Y \leq c$ and $\theta<0$. Rearranging terms in (79) yields

$$
0 \leq m^{\prime}(\theta)-\mu(1+\theta c) m(\theta)
$$

and multiplying each side of (80) by $e^{-\mu\left(\theta+c \theta^{2} / 2\right)}$ we see that

$$
0 \leq\left(m(\theta) e^{-\mu\left(\theta+c \theta^{2} / 2\right)}\right)^{\prime} \quad \text { for all } \theta<0 .
$$

Integrating both sides of (81), and using $m(0)=1$, yields

$$
0 \leq \int_{\theta}^{0}\left(m(x) e^{-\mu\left(x+c x^{2} / 2\right)}\right)^{\prime} d x=1-m(\theta) e^{-\mu\left(\theta+c \theta^{2} / 2\right)}
$$

and hence

$$
m(\theta) \leq e^{\mu\left(\theta+c \theta^{2} / 2\right)}
$$

Next letting $M(\theta)=E\left(e^{\theta(Y-\mu)}\right)=e^{-\mu \theta} m(\theta)$ and applying (82), we obtain the bound

$$
M(\theta) \leq e^{-\mu \theta} e^{\mu\left(\theta+c \theta^{2} / 2\right)}=e^{\mu c \theta^{2} / 2} .
$$

Hence for fixed $t>0$ and all $\theta<0$,

$$
P(Y-\mu \leq-t)=P\left(e^{\theta(Y-\mu)} \geq e^{-\theta t}\right) \leq e^{\theta t} M(\theta) \leq e^{\theta t+\mu c \theta^{2} / 2}
$$

by Markov's inequality. Substituting $\theta=-t / c \mu$ yields inequality (5).

\section{Acknowledgements}

This work was partially supported by NSA grant H98230-11-1-0162 (Bartroff and Goldstein) and NSF grant DMS-1310127 (Bartroff). 


\section{References}

[1] Alon, N. and Spencer, J.H. (2008). The Probabilistic Method, 3rd ed. Wiley-Interscience Series in Discrete Mathematics and Optimization. Hoboken, NJ: Wiley. MR2437651

[2] Andersson, J. (2005). The influence of grain size variation on metal fatigue. Int. J. Fatigue 27 847-852.

[3] Arratia, R. and Baxendale, P. (2015). Bounded size bias coupling: A Gamma function bound, and universal Dickman-function behavior. Probab. Theory Related Fields 162 411-429. MR3383333

[4] Arratia, R., Goldstein, L. and Kochman, F. (2013). Size bias for one and all. Preprint. Available at arXiv:1308.2729.

[5] Azuma, K. (1967). Weighted sums of certain dependent random variables. Tohoku Math. J. (2) 19 357-367. MR0221571

[6] Bagnoli, M. and Bergstrom, T. (2005). Log-concave probability and its applications. Econom. Theory 26 445-469. MR2213177

[7] Barbour, A.D., Karoński, M. and Ruciński, A. (1989). A central limit theorem for decomposable random variables with applications to random graphs. J. Combin. Theory Ser. B 47 125-145. MR1047781

[8] Bartroff, J. and Goldstein, L. (2013). A Berry-Esseen bound for the uniform multinomial occupancy model. Electron. J. Probab. 18 no. 27, 1-29. MR3035755

[9] Boucheron, S., Lugosi, G. and Massart, P. (2013). Concentration Inequalities: A Nonasymptotic Theory of Independence. Oxford: Oxford Univ. Press. MR3185193

[10] Brown, M., Peköz, E.A. and Ross, S.M. (2011). Finding expectations of monotone functions of binary random variables by simulation, with applications to reliability, finance, and round Robin tournaments. In Stochastic Analysis, Stochastic Systems, and Applications to Finance 101-113. Hackensack, NJ: World Sci. Publ. MR2884567

[11] Chao, A., Yip, P. and Lin, H.-S. (1996). Estimating the number of species via a martingale estimating function. Statist. Sinica 6 403-418. MR1399311

[12] Chatterjee, S. (2007). Stein's method for concentration inequalities. Probab. Theory Related Fields 138 305-321. MR2288072

[13] Chen, L.H.Y., Goldstein, L. and Shao, Q.-M. (2011). Normal Approximation by Stein's Method. Probability and Its Applications (New York). Heidelberg: Springer. MR2732624

[14] Chung, F. and Lu, L. (2006). Complex Graphs and Networks. CBMS Regional Conference Series in Mathematics 107. Providence, RI: Amer. Math. Soc. MR2248695

[15] Conway, J.H. and Sloane, N.J.A. (1999). Sphere Packings, Lattices and Groups, 3rd ed. Grundlehren der Mathematischen Wissenschaften [Fundamental Principles of Mathematical Sciences] 290. New York: Springer. MR1662447

[16] Cook, N., Goldstein, L. and Johnson, T. Size biased couplings and the spectral gap for random regular graphs. Ann. Probab. To appear.

[17] Dousse, O., Mannersalo, P. and Thiran, P. (2004). Latency of wireless sensor networks with uncoordinated power saving mechanisms. In Proceedings of the 5th ACM International Symposium on Mobile Ad Hoc Networking and Computing 109-120.

[18] Dubhashi, D. and Ranjan, D. (1998). Balls and bins: A study in negative dependence. Random Structures Algorithms 13 99-124. MR1642566

[19] Efron, B. (1965). Increasing properties of Pólya frequency functions. Ann. Math. Stat. 36 272-279. MR0171335

[20] Efron, B. and Thisted, R. (1976). Estimating the number of unseen species: How many words did Shakespeare know? Biometrika 63 435-448.

[21] Ehm, W. (1991). Binomial approximation to the Poisson binomial distribution. Statist. Probab. Lett. 11 7-16. MR1093412 
[22] Englund, G. (1981). A remainder term estimate for the normal approximation in classical occupancy. Ann. Probab. 9 684-692. MR0624696

[23] Ghosh, S. and Goldstein, L. (2011). Concentration of measures via size-biased couplings. Probab. Theory Related Fields 149 271-278. MR2773032

[24] Ghosh, S. and Goldstein, L. (2011). Applications of size biased couplings for concentration of measures. Electron. Commun. Probab. 16 70-83. MR2763529

[25] Ghosh, S., Goldstein, L. and Raič, M. (2011). Concentration of measure for the number of isolated vertices in the Erdős-Rényi random graph by size bias couplings. Statist. Probab. Lett. 81 1565-1570. MR2832913

[26] Goldstein, L. (2013). A Berry-Esseen bound with applications to vertex degree counts in the ErdősRényi random graph. Ann. Appl. Probab. 23 617-636. MR3059270

[27] Goldstein, L. and Penrose, M.D. (2010). Normal approximation for coverage models over binomial point processes. Ann. Appl. Probab. 20 696-721. MR2650046

[28] Goldstein, L. and Rinott, Y. (1996). Multivariate normal approximations by Stein's method and size bias couplings. J. Appl. Probab. 33 1-17. MR1371949

[29] Hoeffding, W. (1956). On the distribution of the number of successes in independent trials. Ann. Math. Stat. 27 713-721. MR0080391

[30] Hoeffding, W. (1963). Probability inequalities for sums of bounded random variables. J. Amer. Statist. Assoc. 58 13-30. MR0144363

[31] Janson, S. and Nowicki, K. (1991). The asymptotic distributions of generalized $U$-statistics with applications to random graphs. Probab. Theory Related Fields 90 341-375. MR1133371

[32] Joag-Dev, K. and Proschan, F. (1983). Negative association of random variables, with applications. Ann. Statist. 11 286-295. MR0684886

[33] Karoński, M. and Ruciński, A. (1987). Poisson convergence and semi-induced properties of random graphs. Math. Proc. Cambridge Philos. Soc. 101 291-300. MR0870602

[34] Keilson, J. and Gerber, H. (1971). Some results for discrete unimodality. J. Amer. Statist. Assoc. 66 386-389.

[35] Kolchin, V.F., Sevast'yanov, B.A. and Chistyakov, V.P. (1978). Random Allocations. Scripta Series in Mathematics. Washington, DC: V. H. Winston \& Sons. Translated from the Russian, Translation edited by A. V. Balakrishnan. MR0471016

[36] Kordecki, W. (1990). Normal approximation and isolated vertices in random graphs. In Random Graphs '87 (Poznań, 1987) 131-139. Chichester: Wiley. MR1094128

[37] Ledoux, M. (2001). The Concentration of Measure Phenomenon. Mathematical Surveys and Monographs 89. Providence, RI: Amer. Math. Soc. MR1849347

[38] Liggett, T.M. (1985). Interacting Particle Systems. Grundlehren der Mathematischen Wissenschaften [Fundamental Principles of Mathematical Sciences] 276. New York: Springer. MR0776231

[39] Lin, K. and Reinert, G. (2012). Joint vertex degrees in the inhomogeneous random graph model $g\left(n,\left\{p_{i j}\right\}\right)$. Adv. in Appl. Probab. 44 139-165. MR2951550

[40] McDiarmid, C. (1989). On the method of bounded differences. In Surveys in Combinatorics, 1989 (Norwich, 1989). London Mathematical Society Lecture Note Series 141 148-188. Cambridge: Cambridge Univ. Press. MR1036755

[41] McDiarmid, C. and Reed, B. (2006). Concentration for self-bounding functions and an inequality of Talagrand. Random Structures Algorithms 29 549-557. MR2268235

[42] Müller, A. and Stoyan, D. (2002). Comparison Methods for Stochastic Models and Risks. Wiley Series in Probability and Statistics. Chichester: Wiley. MR1889865

[43] Penrose, M.D. (2009). Normal approximation for isolated balls in an urn allocation model. Electron. J. Probab. 14 2156-2181. MR2550296 
[44] Rényi, A. (1962). Three new proofs and a generalization of a theorem of Irving Weiss. Magy. Tud. Akad. Mat. Kut. Intéz. Közl. 7 203-214. MR0148094

[45] Robbins, H.E. (1968). Estimating the total probability of the unobserved outcomes of an experiment. Ann. Math. Stat. 39 256-257. MR0221695

[46] Ross, N. (2011). Fundamentals of Stein's method. Probab. Surv. 8 210-293. MR2861132

[47] Schoenberg, I.J. (1951). On Pólya frequency functions. I. The totally positive functions and their Laplace transforms. J. Anal. Math. 1331-374. MR0047732

[48] Shaked, M. and Shanthikumar, J.G. (2007). Stochastic Orders. Springer Series in Statistics. New York: Springer. MR2265633

[49] Sheridan, G., Jones, O., Nyman, P. and Lane, P. (2011). A stochastic germ-grain model to estimate wildfire induced debris flow risks in a changing climate. American Geophysical Union, Fall Meeting 2011, abstract \#EP31B-0823.

[50] Starr, N. (1979). Linear estimation of the probability of discovering a new species. Ann. Statist. 7 644-652. MR0527498

[51] Stein, C. (1972). A bound for the error in the normal approximation to the distribution of a sum of dependent random variables. In Proc. Sixth Berkeley Symp. Math. Stat. Prob. 583-602. MR0402873

[52] Stein, C. (1986). Approximate Computation of Expectations. Institute of Mathematical Statistics Lecture Notes-Monograph Series 7. Hayward, CA: IMS. MR0882007

[53] Talagrand, M. (1995). Concentration of measure and isoperimetric inequalities in product spaces. Publ. Math. Inst. Hautes Études Sci. 81 73-205. MR1361756

[54] Thisted, R. and Efron, B. (1987). Did Shakespeare write a newly-discovered poem? Biometrika 74 445-455. MR0909350

[55] Weiss, I. (1958). Limiting distributions in some occupancy problems. Ann. Math. Stat. 29 878-884. MR0098422

[56] Zong, C. (1999). Sphere Packings. Universitext. New York: Springer. MR1707318

Received December 2016 and revised May 2017 